KMU FORSCHUNG AUSTRIA

Austrian Institute for SME Research

\title{
Evaluierung des Projektes PROVISO 2003 - 2007
}


Diese Studie wurde im Auftrag des Bundesministeriums für Bildung, Wissenschaft und Kultur (BMBWK) durchgeführt.

KMU FORSCHUNG AUSTRIA

Austrian Institute for SME Research

(Präsident: em. Univ.-Prof. Dr.Dr.h.c. J. Hanns Pichler, M.Sc.)

Für den Inhalt verantwortlich: Walter Bornett

Verfasserlinnen des Berichts:

Sonja Sheikh

Alfred Radauer

Layout:

Susanne Fröhlich

Die vorliegende Studie wurde nach allen Maßstäben der Sorgfalt erstellt.

Die KMU FORSCHUNG AUSTRIA übernimmt jedoch keine Haftung für Schäden oder Folgeschäden die auf diese Studie oder auf mögliche fehlerhafte Angaben zurückgehen. 


\section{INHALTSVERZEICHNIS}

\section{Zusammenfassung}

$1 \quad$ Einleitung 3

2 Analyse der Ausrichtung, Zielgruppen und Datenbasis von PROVISO .......................... 4

$2.1 \quad$ Zielsetzung und Aufgaben von PROVISO ............................................................ 4

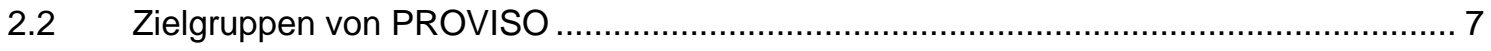

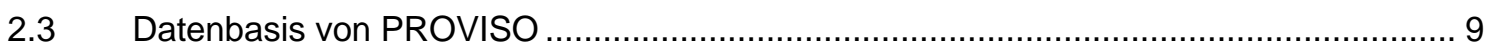

$3 \quad$ Analyse der Produkte und Dienstleistungen von PROVISO ........................................ 10

3.1 Die Produkte und Dienstleistungen von PROVISO im Überblick.................................. 10

3.2 Nutzung der Produkte und Dienstleistungen von PROVISO ....................................... 12

3.3 Zugang zu den Produkten und Dienstleistungen von PROVISO .................................. 16

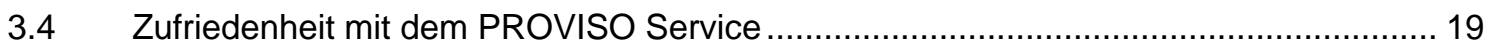

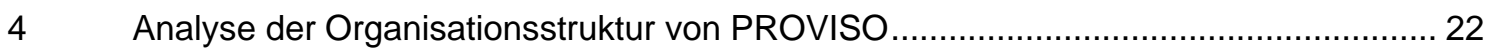

$4.1 \quad$ Organisatorische Einbettung von PROVISO .......................................................... 22

4.2 Interne Organisation der PROVISO Aktivitäten ............................................................... 23

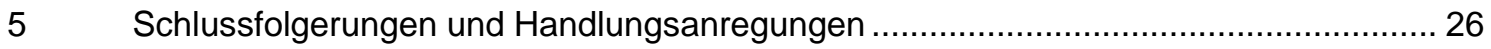

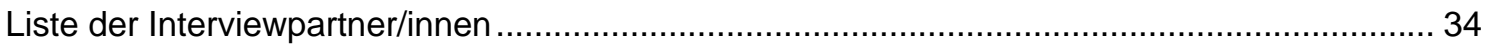

\section{ANHANG}

Die Aufbereitung von Beteiligungsdaten im internationalen Kontext Liste der InterviewpartnerInnen 


\section{Grafikverzeichnis}

Grafik $1 \quad$ Nachfrage nach ROVISO Produkten im Zeitraum 1. September 2003 30. Juni 2005, nach Nutzergruppen, Anteile in Prozent

Grafik 2

Anzahl der erstellten PROVISO Produkte, nach Art des Produkts, 1.

September 2003 - 30. Juni 2005.

Grafik 3 Kenntnis der PROVISO Produkte, nach Zielgruppe, Anteil jener, die das Produkt kennen, in Prozent...

Grafik $4 \quad$ Nutzung der PROVISO Produkte, nach Zielgruppe, Anteil jener, die das Produkt nutzen in Prozent.....

Grafik 5

Kontext der Verwendung der PROVISO Produkte, Anteile in Prozent.

Grafik 6

Relevanz der PROVISO Produkte, nach Zielgruppe, Mittelwerte.

Grafik 7

Bedeutung der PROVISO Produkte und Informationen für unterschiedliche Aktivitäten, nach Zielgruppe, Mittelwerte

Grafik 8

Häufigkeit der Zugriffs auf die PROVISO Homepage, Anteil der

Befragten in Prozent ...

Grafik 9

Bezugsquellen der PROVISO Produkte, Anteil der Befragten in Prozent.

Grafik 10

Bewertung des Zugangs zu PROVISO Produkten, nach Zielgruppe, Anteil in der Zielgruppe in Prozent.

Grafik 11

Zufriedenheit mit unterschiedlichen Aspekten der PROVISO Produkte, nach Zielgruppe, Mittelwerte...

Grafik 12

Zufriedenheit mit dem PROVISO Service, Mittelwerte

\section{Tabellenverzeichnis}

Tabelle 1 Finanzierungsstruktur von PROVISO 2003- 2007 für den Zeitraum 1. September $2003-30$. Juni 2007. 


\section{Zusammenfassung}

Im vorliegenden Bericht werden die Ergebnisse der Evaluierung des Projektes PROVISO 2003 - 2007 (PROVISO IV), die von der KMU FORSCHUNG AUSTRIA im Auftrag des Bundesministeriums für Bildung, Wissenschaft und Kultur (BMBWK) durchgeführt wurde, dargestellt. Für die Evaluierung griff die KMU FORSCHUNG AUSTRIA auf einen Mix aus qualitativen und quantitativen Methoden zurück. Durchgeführt wurden eine Dokumentenanalyse, eine halbstandardisierte schriftliche Befragung bei insgesamt 66 NutzerInnen des Service, 31 qualitative Interviews mit Programmdelegierten, PROVISO MitarbeiterInnen und sonstigen Stakeholdern des Service, sowie ein internationaler Vergleich mit vier kleineren Fallstudien.

PROVISO IV wurde vom BMBWK als federführendem Ressort, dem Bundesministerium für Wirtschaft und Arbeit (BMWA) sowie dem Bundesministerium für Land- und Forstwirtschaft, Umwelt und Wasserwirtschaft (BMLFUW) an den Verein OCG - Österreichische Computer Gesellschaft in Auftrag gegeben mit dem Ziel, ein bestmögliches Monitoring der österreichischen Teilnahme am 6. EU-Rahmenprogramm für Forschung, technologische Entwicklung und Demonstration im internationalen Vergleich sicherzustellen. Damit einhergehend soll PROVISO eine Erfolgskontrolle ermöglichen, Grundlagen für die Steuerung der österreichischen Fördermaßnahmen schaffen und letztlich als Referenz für die Politikformulierung im Hinblick auf strategische europäische Entscheidungs- und Koordinationsprozesse dienen.

Primäre Zielgruppe von PROVISO sind die österreichischen Programmdelegierten und die EUKoordinationsabteilung des BMBWK. Eine weitere Zielgruppe, die im Vergleich zu früheren PROVISO Projekten deutlich an Bedeutung gewonnen hat, sind die FFG - Österreichische Forschungsförderungsgesellschaft, Bereich Europäische und Internationale Programme sowie die regionalen Beratungs- und Betreuungszentren (RBBZ) als Betreuungseinrichtungen zum 6. EU-Rahmenprogramm. Andere Organisationen können sich ebenfalls mit Anfragen an PROVISO wenden, wobei diese Anfragen nach Maßgabe der zur Verfügung stehenden Ressourcen unter Berücksichtigung der von der EU-Kommission den Delegierten auferlegten Vertraulichkeitsbestimmungen bearbeitet werden.

Das PROVISO Service lässt sich in zwei Komponenten teilen: Im Rahmen der MonitoringKomponente werden die Beteiligung österreichischer ForscherInnen am 6. EU Rahmenprogramm erfasst und die Grundlagen für eine Erfolgskontrolle für die Steuerung von Fördermaßnahmen und die Politikformulierung geschaffen. Hierzu übermitteln die Programmdelegierten Beteiligungsdaten, wie sie innen von der Kommission auf den CIRCA Servern zur Verfügung gestellt werden, an PROVISO, das diese aufbereitet, standardisiert und korrigiert und in eine ACCESS Datenbank einspielt. Die Service-Komponente beinhaltet darauf aufbauend die Erstellung von Analysen und Auswertungen, für die Zielgruppen von PROVISO. Dabei stehen neben allgemeinen Informationen und Auskünften sowie sog. „Spezialauswertungen“ folgende regelmäßig und periodisch erstellte Produkte zur Verfügung: Callinformationen/Schnellauswertungen (zur Lieferung eines aktuellen Kurzüberblicks über Ergebnisse der Ausschreibungen) und Status Reports (Folienübersichten über den aktuellen Stand der österreichischen Beteiligung am 6. EU Rahmenprogramm). Fakultative Produkte, die ausschließlich auf Anfrage bei Bedarf erstellt werden, sind Präsentationsmaterialien, Thematische Dossiers (themenspezifische Auswertungen, in die auch andere nationale oder internationale FTE- und Wirtschaftsdaten einfließen), Programm Reports und Jahres Reports.

In organisatorischer Hinsicht ist das PROVISO Team ähnlich einer Stabsstelle in die Ministerialstruktur des BMBWK eingebunden. Für die Erstellung der Produkte ist ein Team von insgesamt 7 Personen verantwortlich, wovon vier Vollzeit und drei Teilzeit (im Ausmaß von 20 Stunden pro Woche) tätig sind. Die Einbindung in die Ministerialstruktur hat vor allem den Vorteil kurzer Kommunikationswege zur primären Zielgruppe von PROVISO, nämlich den Programmdelegierten. Demgegenüber dürften jedoch Synergieeffekte mit der Zielgruppe der Betreuungseinrichtungen vielfach ungenutzt bleiben. 
Der Größe der Serviceeinrichtung und der Nähe zu den Delegierten entsprechend kommt der informellen und persönlichen Kommunikation innerhalb und mit PROVISO eine hohe Bedeutung zu. Der Großteil der Anfragen (mehr als $52 \%$ ) wird direkt an PROVISO gerichtet, wobei das PROVISO Team die Produkte im Anschluss per E-Mail versendet. Elektronische Verbreitungskanäle, wie z.B. era.gv.at oder die Projektmanagementplattform EPMP, sind im Vergleich weniger wichtig. Dies gilt auch für die PROVISO Homepage, die zwar $74 \%$ der Nutzerlnnen kennen, aber nur selten nutzen, da die entsprechende Informationsübermittlung meist persönlich bzw. über E-Mail erfolgt. Insgesamt wird der Zugang zu den PROVISO Informationen von den NutzerInnen überwiegend als leicht eingestuft.

Die PROVISO Produkte sind innerhalb der Zielgruppe von PROVISO weitestgehend gut bekannt. Damit einher geht auch eine vergleichsweise hohe Nutzung, wobei deutlich wird, dass die Beraterlnnen der FFG bzw. der RBBZ die Produkte genauso intensiv bzw. zum Teil sogar intensiver nutzen als die Programmdelegierten. Die Ausweitung der Zielgruppe um die Betreuungseinrichtungen hat somit einen wichtigen Beitrag zur effektiveren Verwendung von PROVISO geleistet. Die meisten NutzerInnen bezeichnen die PROVISO Produkte als für ihre Arbeit relevant oder sehr relevant, wobei die Betreuungseinrichtungen (aber auch die externen NutzerInnen) die Bedeutung des Services im Durchschnitt sogar höher einschätzen als die Programmdelegierten. Wichtig ist PROVISO für die Betreuungseinrichtungen vor allem in Hinblick auf die Durchführung von Beratungsgesprächen.

Generell besteht eine hohe Zufriedenheit mit den PROVISO Produkten und Dienstleistungen seitens der unterschiedlichen Zielgruppen. Verbesserungspotenziale sind primär in Teilbereichen erkennbar, wie beispielsweise in Bezug auf die Klarheit der Erläuterungen oder die Übersichtlichkeit der Informationen. Hinsichtlich der Verwendbarkeit und der Qualität der Produkte wird kritisiert, dass viele Analysen eine inhaltlich fundierte Auseinandersetzung mit den Daten seitens der PROVISO MitarbeiterInnen missen lassen. Vorgeschlagen wird hier unter anderem eine stärkere Einbindung der Programmdelegierten in die Interpretation der Daten.

Insgesamt zeigen die Evaluationsergebnisse, dass PROVISO ein wertvolles Asset für die Strategieentwicklungs- und Beratungstätigkeit der Zielgruppen in Zusammenhang mit dem 6 . EU Rahmenprogramm sowie für die Erfolgskontrolle der österreichischen Beteiligung darstellt. Verbesserungspotenziale des Service liegen eher im Detail bzw. betreffen die zukünftige Positionierung von PROVISO. Die Schlussfolgerungen und Handlungsempfehlungen sind in Kapitel 5 des Berichtes dargestellt. 


\section{Einleitung}

PROVISO 2003 - 2007 ist ein Projekt, welches von der Republik Österreich, vertreten durch das Bundesministerium für Bildung, Wissenschaft und Kultur (BMBWK) als federführendes Ressort, das Bundesministerium für Wirtschaft und Arbeit (BMWA) sowie das Bundesministerium für Land- und Forstwirtschaft, Umwelt und Wasserwirtschaft (BMLFUW) mit Vertrag vom 4. August 2003 an den Verein OCG - Österreichische Computer Gesellschaft in Auftrag gegeben wurde. Seit Juli 2004 beteiligt sich auch das Bundesministerium für Verkehr, Innovation und Technologie (BMVIT) an PROVISO. PROVISO definiert sich dabei als eine wissensbasierte Dienstleistungs- und Informationsstruktur, die den zuständigen Ressorts eine professionelle Informationsinfrastruktur für die politische Strategie- und Maßnahmenentwicklung sowie für die Öffentlichkeitsarbeit im Bereich der FTE Politik der Europäischen Union und insbesondere in Hinblick auf das 6. EU Rahmenprogramm für Forschung, technologische Entwicklung und Demonstration bieten soll.

Der Vertrag zwischen den beteiligten Ministerien und der OCG sieht eine Evaluierung der Tätigkeiten von PROVISO im Jahr 2005 vor. Darüber hinaus hat das PROVISO Projekt seit der knapp 10 Jahre seines Bestehens einige - sowohl organisatorische als auch inhaltliche Veränderungen erfahren, die eine Analyse der Positionierung von PROVISO sowohl nach innen (Zielsetzung und Ausrichtung von PROVISO, Einbettung in die Ministerialstruktur, Schnittstelle zu den Programmdelegierten etc.) als auch nach außen (Kooperation mit den Zielgruppen, Wahrnehmung in der Öffentlichkeit etc.) rechtfertigen. Vor diesem Hintergrund hat das BMBWK die KMU FORSCHUNG AUSTRIA mit der Evaluierung des Projektes PROVISO 2003 - 2007 beauftragt. Zielsetzung der Evaluierung ist die Überprüfung der Effektivität und Effizienz des PROVISO Service sowie die Schaffung von Grundlagen zu dessen Optimierung.

Die Evaluierung beruht sowohl auf quantitativen als auch qualitativen Analysen unterschiedlicher Aspekte des PROVISO Service. Im Rahmen von zwei schriftlichen Fragebogenerhebungen im Zeitraum Juli / September 2005 wurden sowohl die unmittelbaren Zielgruppen von PROVISO (d.s. die Programmdelegierten der spezifischen Programme des 6. EU-Rahmenprogramms, die ständigen ExpertInnen zum 6. EU-Rahmenprogramm, die Abteilung für EUForschungspolitik und Koordination des BMBWK sowie die BeraterInnen der FFG - Österreichische Forschungsförderungsgesellschaft, Bereich Europäische und Internationale Programme und der regionalen Beratungs- und Betreuungszentren (RBBZ) für das 6. EU-Rahmenprogramm) als auch externe Nutzer von PROVISO (in erster Linie Forschungsgruppen und Organisationen, die in der Vergangenheit Anfragen an PROVISO gestellt haben) zu Ihren Erfahrungen mit dem Service befragt. Darüber hinaus wurden insgesamt 31 Interviews mit den Auftraggebern und Mitarbeiterlnnen von PROVISO, Programmdelegierten, BeraterInnen der FFG/RBBZ und externen Stakeholdern und Experten durchgeführt.

Die Ergebnisse der Analysen sind in den folgenden Kapiteln dargestellt. Kapitel 2 enthält eine Analyse der Ausrichtung, Zielgruppen und Datenbasis von PROVISO, in Kapitel 3 werden die Produkte und Dienstleistungen von PROVISO, basierend auf den durchgeführten Befragungen analysiert und Kapitel 4 enthält eine kurze Analyse der Organisationsstruktur von PROVISO. In Kapitel 5 sind schließlich einige Schlussfolgerungen und Handlungsempfehlungen basieren auf den durchgeführten Erhebungen und Analysen dargestellt. Darüber hinaus befinden sich im Anhang die Ergebnisse einer kleinen internationalen Recherche in Bezug auf die Aufbereitung von Beteiligungsdaten zu den EU-Rahmenprogrammen im internationalen Kontext. 


\section{Analyse der Ausrichtung, Zielgruppen und Datenbasis von PROVISO}

\subsection{Zielsetzung und Aufgaben von PROVISO}

Für die Analyse der Zielsetzung und heutigen Aufgaben von PROVISO scheint ein kurzer Rückblick auf die Entstehungsgeschichte von PROVISO hilfreich. Die Basis für das heutige PROVISO Projekt wurde in der PROVISO-Aufbauphase 1996 - 1997 durch die Betreuung der Programme ESPRIT und BRITE/ EURAM im 4. EU-Rahmenprogramm gelegt. Die Delegierten in den Programmkomitees der einzelnen Programme erhielten eine Vielzahl an Daten und Informationen von der EU-Kommission. Gleichzeitig waren diese Daten und Informationen häufig fehlerhaft und in ihrer Struktur inkonsistent. Motiv und Zielsetzung des damaligen PROVISO Projekts (PROVISO I) war es daher, die Daten in (eine) Ordnung zu bringen und, darauf aufbauend, Analysen durchzuführen und diese zu interpretieren, um die Programmdelegierten in ihrer täglichen Arbeit zu unterstützen und Stärken und Schwächen der österreichischen Beteiligung in den einzelnen Programmbereichen erkennen zu können. ${ }^{1}$

Im Laufe der PROVISO Tätigkeit zeigte sich die Notwendigkeit, weitere Programme in das System mit einzubeziehen, um einen vollständigen Überblick über die Beteiligung Österreichs an den EU-Rahmenprogrammen zu erhalten. Von 1998 bis Juni 2000 wurde PROVISO damit auf den Betreuungsumfang des 5. EU-Rahmenprogramms erweitert (PROVISO II). Neben der Datenaufbereitung und -analyse wurden damals von den PROVISO Mitarbeiterlnnen auch klassische Assistenzaufgaben für die Programmdelegierten wahrgenommen, wie etwa die Vorbereitung der Programmkomiteesitzungen in Brüssel, die Beschaffung und Aufbereitung von Hintergrundinformationen, die Vorbereitung von Veranstaltungen etc. Jeder/m Programmdelegierte/n war ein/e PROVISO Mitarbeiterln zugeordnet, der/die zu etwa $50 \%$ PROVISO Tätigkeiten und zu 50 \% Assistenztätigkeiten durchführen sollte. Die PROVISO MitarbeiterInnen waren räumlich in den Ressorts der jeweiligen Programmdelegierten angesiedelt.

1999 kam es dann zu einem Wechsel des Projektträgers von PROVISO, das von dem damaligen Forschungszentrum Seibersdorf an die Österreichische Computer Gesellschaft (OCG) überging (PROVISO III). Im Jahr 2000 erfolgte die Aufspaltung des bis dahin für PROVISO zuständigen Bundesministeriums für Wissenschaft und Verkehr (BMWV) in das heutige Bundesministerium für Bildung, Wissenschaft und Kultur (BMBWK) und das Bundesministerium für Verkehr, Innovation und Technologie (BMVIT). Die PROVISO MitarbeiterInnen waren nun auf mehrere Standorte verteilt.

Im Jahr 2002/2003 stand PROVISO vor einer Reihe struktureller Veränderungen, die den Grundstein für das heutige PROVISO 2003 - 2007 (PROVISO IV) legten. Das BMVIT zog sich von PROVISO zurück (beteiligt sich jedoch seit Juni 2004 wieder über einen Sondervertrag an PROVISO), gleichzeitig übernahm das BMBWK die Federführung für PROVISO und führte alle Standorte zu einem einzigen innerhalb des BMBWK zusammen. Mit der Neustrukturierung von PROVISO entfiel auch die Assistenztätigkeit durch die PROVISO MitarbeiterInnen. Die Projektkosten für das heutige PROVISO betragen für den Beauftragungszeitraum 1. September 2003 - 30. Juni 2007 insgesamt $€ 1$ 1.313.806,- (siehe Tabelle 1). Der Großteil der Kosten $(65,6 \%)$ wird hierbei vom BMBWK getragen, gefolgt vom BMVIT $(21,8 \%)$, dem BMLFUW $(8,2 \%)$ und dem BMWA $(4,1 \%)$.

Topolnik, M., Schibany, A. (1997): Evaluierung der Beteiligung an FTE-Programmen der Europäischen Union: Ein Leitfaden, Seibersdorf Report, Wien, September 1997 
Finanzierungsstruktur von PROVISO 2003 - 2007 für den Zeitraum 1. September 2003 - 30. Juni 2007

\begin{tabular}{l|c|c}
\hline \multicolumn{1}{c|}{ Ressort } & $\begin{array}{c}\text { maximaler } \\
\text { Finanzierungsbeitrag in } €\end{array}$ & Finanzierungsanteil in \% \\
\hline BMBWK & $864.903,-$ & 65,8 \\
BMLFUW & $108.152,-$ & 8,2 \\
BMWA & $54.024,-$ & 4,1 \\
BMVIT $\left.^{\star}\right)$ & $286.727,-$ & 21,8 \\
\hline \hline Gesamt & $1.313 .806,-$ & 100,0 \\
\hline${ }^{*}$ Das BMVIT ist erst seit 30.06.2004 an PROVISO 2003 - 2007 beteiligt \\
Quelle: Vertrag zwischen der Republik Österreich, vertreten durch das BMBWK, als federführendes Ressort, das \\
\multicolumn{2}{c}{ BMWA und das BMFLUW sowie der OCG - Österreichische Computer Gesellschaft (GZ.: 360.058/2-VI/5/2003) } \\
\hline
\end{tabular}

Das Ziel des heutigen PROVISO Information Service 2003 - 2007 besteht in der Sicherstellung eines bestmöglichen Monitorings der österreichischen Teilnahme am 6. EU-Rahmenprogramm für Forschung, technologische Entwicklung und Demonstration im internationalen Vergleich, mit der Zielsetzung der Erfolgskontrolle, der Schaffung von Grundlagen für die Steuerung der österreichischen Fördermaßnahmen sowie einer Referenz für die Politikformulierung im Hinblick auf strategische europäische Entscheidungs- und Koordinationsprozesse. ${ }^{2}$

Als Aufgabenbereiche von PROVISO 2003 - 2007 sind laut Vertrag zwischen der Republik Österreich, vertreten durch das BMBWK, als federführendes Ressort, das BMWA und das BMLFUW sowie der OCG - Österreichische Computer Gesellschaft folgendermaßen definiert:

- kontinuierliche Datenerfassung und Dokumentation;

- Harmonisierung der nationalen und internationalen Daten sowie Gewährleistung einer benutzerfreundlichen Handhabung;

- Rascher Überblick über die Performance Österreichs in den Ausschreibungen;

- graphische Aufbereitung der Daten und Informationen;

- die Bereitstellung einer kompatiblen Datenbasis und Informationsstruktur;

- inhaltlich thematische Analysen der österreichischen Forschung in den EU-Rahmenprogrammen im Speziellen und der europäischen Forschung im Allgemeinen.

Damit lässt sich PROVISO 2003 - 2007 im Wesentlichen in zwei Komponenten zerlegen: ${ }^{3}$

1. Die Monitoring-Komponente: Diese umfasst das Monitoring und die Erfolgskontrolle der österreichischen Beteiligung am 6. EU-Rahmenprogramm für Forschung, technologische Entwicklung und Demonstration als Grundlage für die Steuerung von Fördermaßnahmen und die Politikformulierung.

2. Die Service-Komponente: Diese umfasst die Erarbeitung von Auswertungen und Analysen für die Programmdelegierten als Grundlage für ihre steuerungspolitischen Managementaufgaben sowie für die anderen Zielgruppen von PROVISO.

Vertrag zwischen der Republik Österreich, vertreten durch das BMBWK, als federführendes Ressort, das BMWA und das BMFLUW sowie der OCG - Österreichische Computer Gesellschaft (GZ.: 360.058/2-VI/5/2003)

3 Die „künstliche“ Zerlegung von PROVISO in diese beiden Komponenten erfolgt nur, um die Argumentation im Folgenden besser strukturieren zu können, tatsächlich sind diese beiden Komponenten von PROVISO sehr eng miteinander verknüpft und lassen sich in der täglichen Arbeit nur schwer voneinander trennen. 
In Hinblick auf die Monitoring-Komponente hat PROVISO in den vergangenen Jahren, insbesondere durch eine kontinuierliche Verbesserung seiner Produkte und Dienstleistungen sowie die Ausweitung auf alle relevanten Programme des 6. EU-Rahmenprogramms eine deutliche Weiterentwicklung und Professionalisierung erfahren, die eine wichtige Grundlage für ein umfassendes Technologie- und Forschungsmonitoring darstellen. Vor allem auf einer programmübergreifenden Ebene erfüllt PROVISO damit wichtige Anforderungen seitens der Auftraggeber, und insbesondere seitens der EU-Koordinationsabteilung, und stell diesen eine fundierte Informationsbasis für die Strategieentwicklung (auf nationaler und internationaler Ebene) und die Politikberatung zur Verfügung.

In Hinblick auf die Service-Komponente für die Programmdelegierten wird die Funktion von PROVISO hingegen durchaus kritisch beurteilt. Für die einzelnen Fachabteilungen hat PROVISO als Serviceleistung im Laufe der Zeit deutlich an Bedeutung verloren. Dies wird zum einen damit begründet, dass sich PROVISO in den Augen der Programmdelegierten zunehmend in Richtung Monitoringinstrument für die EU-Koordinationsabteilung entwickelt hat, was - nach eigenen Angaben - zum Teil auch eine entsprechende Zurückhaltung in der Nutzung von PROVISO Leistungen bewirkt, zum anderen sehen die Programmdelegierten eine Einschränkung der Service-Funktion durch den Wegfall der im 5. EU-Rahmenprogramm noch ergänzend zu PROVISO verfügbaren Assistenzleistungen (siehe oben). Kritisch ist diese Entwicklung vor allem aus zwei Gründen zu sehen: zum einen leistet sie der Entstehung von Parallelstrukturen Vorzug - beispielsweise der Schaffung eigener Datenbanken - zum anderen wird damit das Potenzial von PROVISO nicht vollständig ausgeschöpft, was den enormen Ressourcenaufwand für die Datenaufbereitung und -bereinigung in Frage stellt und eine effektive Nutzung von PROVISO verhindert.

In diesem Zusammenhang scheint v.a. auch eine klarere und transparentere Definition und vor allem Kommunikation der Aufgaben und Leistungen von PROVISO, insbesondere gegenüber den Programmdelegierten notwenig. „Wir bräuchten hin und wieder Spezialauswertungen, aber dafür ist PROVISO eh nicht zuständig bzw. hat auch keine Zeit dazu“ war eine nicht selten gehörte Aussage in den Interviews mit den Programmdelegierten. Diese richten sich in erster Linie mit Standardauswertungen bzw. -anfragen an PROVISO, während das breite Feld der inhaltlich themenspezifischen Analysen - zumindest seitens der Programmdelegierten - weitestgehend unausgeschöpft bleibt. Gerade hierin wird jedoch ein hohes Potenzial bzw. ein hoher Nutzen des PROVSIO Service gesehen. 


\subsection{Zielgruppen von PROVISO}

Die unmittelbaren Zielgruppen von PROVISO 2003 - 2007 laut Vertrag sind:

- die zuständigen Programmdelegierten der spezifischen Programme des 6. EU-Rahmenprogramms;

- $\quad$ die Abteilung für EU-Forschungspolitik und Koordination des BMBWK (als hauptverantwortliches Ressort für das 6. EU-Rahmenprogramm);

- die ständigen ExpertInnen zum 6. EU-Rahmenprogramm (im Auftrag der beteiligten Ressorts, vom Auftraggeber zu benennen).

Auf Wunsch des BMBWK kann PROVISO auch externen Forschungsgruppen und Organisationen Auswertungen und Informationen zur Verfügung stellen. ${ }^{4}$

Darüber hinaus erging im Jänner 2005 ein Sonderschreiben des BMBWK an die FFG Österreichische Forschungsförderungsgesellschaft, Bereich Europäische und Internationale Programme sowie an die regionalen Beratungs- und Betreuungszentren für das 6 . EURahmenprogramm (APS - European Programms for Technologies and Training, BEP - Büro für Europäische Programme, CATT- Innovation Management GmbH sowie ITG - Innovationsund Technologietransfer Salzburg $\mathrm{GmbH}$ ), die damit erstmals auch PROVISO Leistungen (z.B. Daten der Projekte mit österreichischer Beteiligung sowie Schnellauswertungen) erhalten.

Die vorrangige Zielgruppe von PROVISO bilden jedoch die österreichischen Programmdelegierten. Etwa $60 \%$ der 480 im Zeitraum 1. September 2003 bis 30. Juni 2005 erstellten PROVISO Produkte wurden für die Delegierten erstellt (siehe Grafik 1). $6 \%$ der PROVISO Produkte wurden von der Abteilung für EU-Forschungspolitik und Koordination des BMBWK beauftragt.

Grafik $1 \quad$ Nachfrage nach PROVISO Produkten im Zeitraum 1. September 2003 - 30. Juni 2005, nach Nutzergruppen, Anteile in Prozent *)

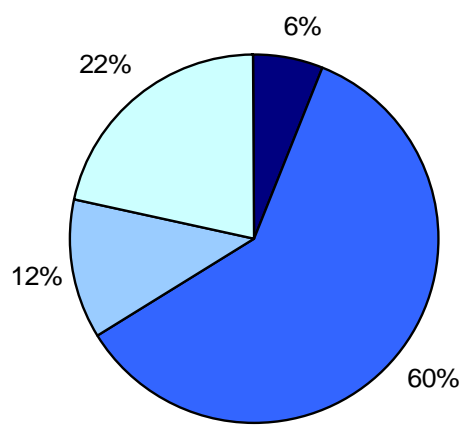

EU-Koordinationsabteilung $\square$ Programmdelegierte $\square$ FFG-EP/RBBZ $\square$ externe Nutzer Innen

*) geschätzte Werte

Quelle: 1. Projektfortschrittsbericht (PBpro734top011004) Oktober 2004 sowie Sonderauswertung September 2005 mit vorläufigen Werten, eigene Berechnungen

4 Vertrag zwischen der Republik Österreich, vertreten durch das BMBWK, als federführendes Ressort, das BMWA und das BMFLUW sowie der OCG - Österreichische Computer Gesellschaft (GZ.: 360.058/2-VI/5/2003) 
Eine zunehmende Bedeutung erlangen externe Forschungsgruppen und Organisationen als Nachfrager und Nutzer von PROVISO Leistungen (siehe auch Kapitel 3), von welchen innerhalb des Betrachtungszeitraums $22 \%$ der Anfragen stammen. Zwar stellen diese keine primäre Zielgruppe von PROVISO dar - externe Anfragen werden in Abhängigkeit der vorhandenen Ressourcen bei PROVISO bearbeitet - jedoch hat die Zahl der Anfragen an PROVISO durch externe Forschungsgruppen und Organisationen in der Vergangenheit deutlich zugenommen. Zusätzliche Anfragen stammen dabei vor allem von den Zielgruppen des 6. EU-Rahmenprogramms (Universitäten, Forschungseinrichtungen) und deren Repräsentanten (Rektorenkonferenz, Interessensvertretungen) sowie seitens der österreichischen Forschungsförderungsfonds. Eine Steigerung an Anfragen zeigt sich auch aus dem Bereich der außeruniversitären Forschungseinrichtungen, die sich in der jüngeren Vergangenheit vor allem in Zusammenhang mit den durch die Ressorts beauftragten Studien (z. B. Evaluierung von FIT IT, Evaluierung der österreichischen Betreuungsstrukturen für das 6. EU-Rahmenprogramm) ergab. Insgesamt wurden von PROVISO im analysierten Zeitraum alle externen Anfragen im Rahmen der vorhandenen Ressourcen bearbeitet.

Mit einem Sonderschreiben des BMBWK an den Bereich Europäische und Internationale Programme der FFG sowie die regionalen Beratungs- und Betreuungszentren für das 6 . EU Rahmenprogramm (RBBZ) ist eine weitere wichtige Zielgruppe von PROVISO hinzugekommen. ${ }^{5} 12 \%$ der PROVISO Produkte wurden im Zeitraum 1. September 2003 bis 30. Juni 2004 für die FFG oder die RBBZ erstellt. 


\subsection{Datenbasis von PROVISO}

Die wichtigsten beiden Säulen von PROVISO stellen die PROVISO Datenbank sowie die auf den Daten basierenden Auswertungen und Analysen der österreichischen Beteiligung an den EU-Rahmenprogrammen dar. Bei der PROVISIO Datenbank handelt es sich um eine Accessdatenbank, die im Wesentlichen die Antrags- bzw. Programmstruktur des 6. EU Rahmenprogramms widerspiegelt.

Datenbasis für PROVISO bilden die Rohdaten der Europäischen Kommission über die Teilnahme am 6. EU-Rahmenprogramm, die diese den jeweiligen Programmdelegierten über den CIRCA Server übermittelt. Alle Programmdelegierten haben Zugriff auf CIRCA und leiten die dort zur Verfügung gestellten Daten an PROVISO weiter. Erfasst werden von PROVISO dabei Informationen (Projektkosten, Finanzierung, Laufzeit, Programmbereiche, inhaltliche Schwerpunkte etc.) zu allen Projektvorschlägen und laufenden Projekten sowie zu den Beteiligungen am 6. EU-Rahmenprogramm aus allen Ländern. Darüber hinaus werden neben den österreichischen Partnern in den jeweiligen Projekten auch alle internationalen Koordinatoren und Partner sowie die entsprechend Informationen (z. B. Land, Organisationstyp, Partnerkosten und -finanzierung etc.) aufgenommen. Die von den Delegierten zur Verfügung gestellten Daten werden von den PROVISO-MitarbeiterInnen - auf Grund der unterschiedlichen und z. T. inkohärenten Datenstruktur - entsprechend bereinigt und in die vorliegende Struktur der PROVISO Datenbank eingespielt. Mit der im Juli 2004 stattgefundenen Erweiterung um jene Programmbereiche, für die das BMVIT zuständig ist, umfasst das PROVISO Service heute alle Programme des 6. EU-Rahmenprogramms. ${ }^{6}$

Insbesondere im Übergang vom 5. zum 6. EU-Rahmenprogramm hat sich dabei die Qualität der von der EU-Kommission gelieferten Daten deutlich verschlechtert. Die Daten werden immer häufiger im pdf- oder Word-Format geliefert und müssen von PROVISO-MitarbeiterInnen erst in ein verarbeitbares Datenformat gebracht werden, bevor sie für weitere Analysen zur Verfügung stehen. ${ }^{7}$ Darüber hinaus weist das 6. EU-Rahmenprogramm eine heterogenere Programmstruktur im Vergleich zum 5. EU-Rahmenprogramm auf (z. B. höhere Anzahl an kleineren Ausschreibungen, ${ }^{8}$ Veränderungen der Sub-Strukturen innerhalb einzelner Programme im Zeitverlauf etc.). Durch diese Entwicklungen hat der Prozess der Datenaufbereitung und -bereinigung im 6. EU-Rahmenprogramm innerhalb des Tätigkeitsspektrums von PROVISO an Bedeutung gewonnen. Wurden beispielsweise im 5. EU-Rahmenprogramm noch etwa $20 \%$ der Ressourcen von PROVISO für die Datenaufbereitung verwendet, so sind dies im 6. EU-Rahmenprogramm - trotz Einsatzes moderner Technologien wie Texterkennungsprogrammen und Scannern - etwa $30 \%$. Zur Aufbereitung dieser Daten werden von PROVISO z. T. externe Kräfte auf Werkvertragsbasis herangezogen (siehe auch Kapitel 4.2).

Ein weiteres Problem in Zusammenhang mit den von der EU-Kommission gelieferten Daten besteht im Bereich des Datenschutzes. Die Programmdelegierten verpflichten sich gegenüber der EU-Kommission zur Geheimhaltung der innen zur Verfügung gestellten Daten. Diese ist jedoch von Programm zu Programm unterschiedlich geregelt und wird auch von den Programmdelegierten unterschiedlich „streng“ gehandhabt. Generell stellt die interne Weitergabe der Daten an das PROVISO Service kein Problem dar, PROVISO-Anfragen beispielsweise seitens der BeraterInnen der FFG bzw. der RBBZ müssen jedoch häufig an die Programmdelegierten gerichtet werden, welche diese dann an das PROVISO Service weiter leiten. Darüber hinaus sind Aspekte in Zusammenhang mit dem Datenschutz auch bei der Weitergabe der Daten durch PROVISO an Dritte, beispielsweise an externe Forschungsgruppen und Organisationen, zu berücksichtigen, insbesondere dann, wenn es sich um Auswertungen auf einzelbetrieblicher Ebene handelt.

6 Für das Programm Innovation werden nur die Eckdaten in der PROVISO Datenbank erfasst, jedoch keine Servicefunktion für den entsprechende Programmdelegierten angeboten.

7 Von einzelnen Akteuren wird vermutet, dass die EU-Kommission die Daten bewusst in einem nicht oder nur schwer weiter zu verarbeitendem Format liefert, um den Aufbau nationaler Datenbanken als „Konkurrenzprodukt“ zu den EU-Datenbanken bzw. das Durchführen sog. nationaler „Rückflussrechnungen“ zu erschweren.

8 Im gesamten 5. EU-Rahmenprogramm wurden von PROVISO etwa 100 Ausschreibungen bearbeitet, zur Halbzeit des 6. EU-Rahmenprogramms waren es bereits 250 . 


\section{Analyse der Produkte und Dienstleistungen von PROVISO}

\subsection{Die Produkte und Dienstleistungen von PROVISO im Überblick}

Auf Basis der Daten in der PROVISO Datenbank werden Auswertungen und Analysen der österreichischen Beteiligung an den EU-Rahmenprogrammen vorgenommen, die von PROVISO in Form unterschiedlicher Produkte und Dienstleistungen zur Verfügung gestellt werden. Dabei ist zu differenzieren zwischen Standardprodukten, die regelmäßig erstellt und an die jeweiligen Adressaten übermittelt werden, und fakultativ zu wählenden Produkten, die je nach Bedarf auf Anfrage erstellt werden:

\section{Standardprodukte:}

- Callinformationen/Schnellauswertungen: Kurzüberblick über Ergebnisse der Ausschreibungen inklusive Listen mit Angaben zu Projekten mit österreichischer Beteiligung am 6. EU-Rahmenprogramm und österreichischen Partnern;

- $\quad$ Status Reports: Folienübersichten über den aktuellen Stand der österreichischen Beteiligung am 6. EU-Rahmenprogramm, programmübergreifend (Folien oder Bericht zweimal jährlich anlässlich der EFF Präsentation);

Fakultative Produkte:

- Präsentationsmaterial: Folien u. ä.;

- $\quad$ Auskünfte / Informationen: Materialien zu kurzfristigen internen und externen Anfragen;

- Thematische Dossiers: Thematische Auswertungen zu programmspezifischen oder programmübergreifenden Themenschwerpunkten, Teilnehmergruppen etc. unter Einbeziehung vorhandener und kostenlos zugänglicher nationaler / internationaler FTE- oder Wirtschaftsdaten;

- Programm-Reports: Kumulierte Gesamtauswertungen, nach Bedarf als Midterm-Bericht bzw. nach Abschluss der Programme.

- Jahres Reports

Insgesamt wurden im Zeitraum 1. September 2003 - 30. Juni 2005480 PROVISO Produkte erstellt (siehe auch Kapitel 2.2). Den Großteil der Produkte machten dabei Auskünfte/allgemeine Informationen aus: 206 derartige Anfragen wurden im Betrachtungszeitraum an PROVISO gerichtet. Besonders bedeutsam waren zudem Callinformationen/Schnellauswertungen, die als Grundlage für die Programmausschusssitzungen dienen und insgesamt 140 Mal erstellt wurden. Des Weiteren wurden 66 Folien/Präsentationen, 10 Thematische Dossiers, 5 Status und 2 Programm Reports verfasst. Sonstige Produkte (wie z.B. Jahres Reports, aber auch Anfragen, die keiner der aufgelisteten Produktarten entspricht), entsprechen 12,5\% der Anfragen (bzw. 51 Anfragen). 
Grafik 2 Anzahl der erstellten PROVISO Produkte, nach Art des Produkts, 1. September 2003 - 30. Juni 2005

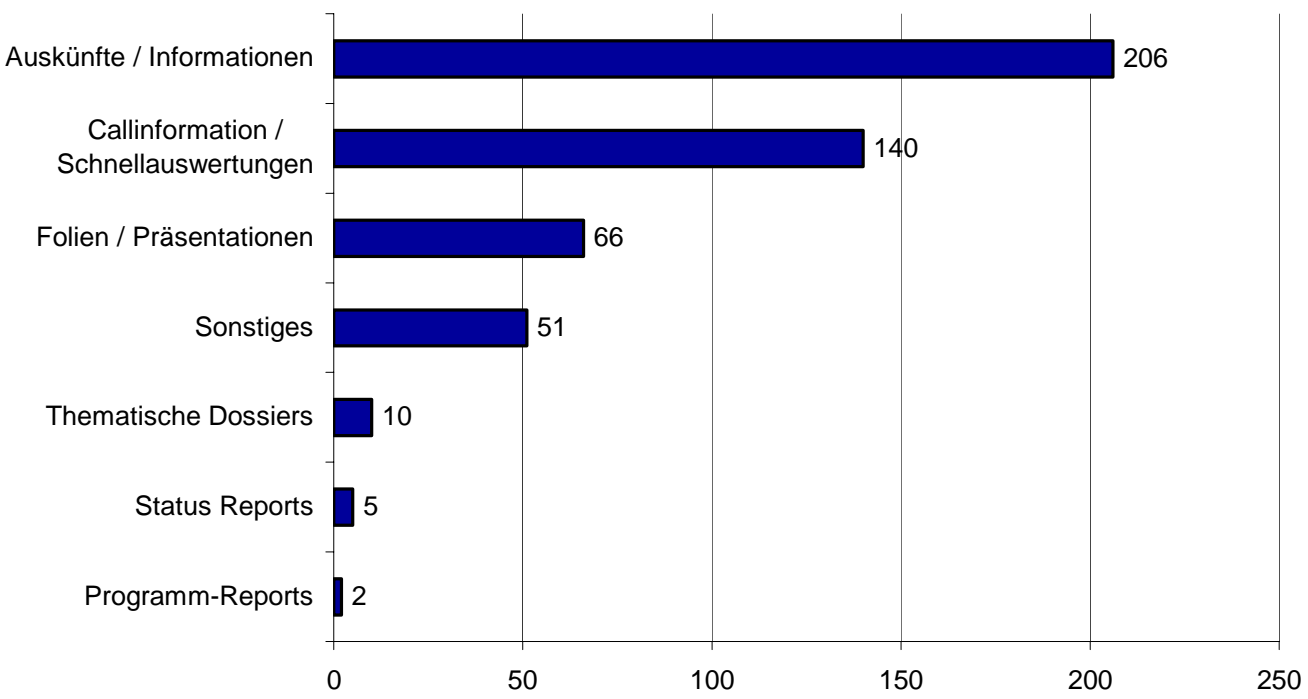

Quellen: 1. Projektfortschrittsbericht (PBpro734top011004) Oktober 2004 sowie Sonderauswertung September 2005 mit vorläufigen Werten

Am häufigsten wurden PROVISO Produkte innerhalb des Programms Mobility (HRM) nachgefragt, gefolgt von den Programmen Food Quality and Safety (FOOD) und Nanotechnologies and -sciences, Intelligent Materials, and New Products (NMP). Vergleichsweise niedrig ist die Inanspruchnahme der PROVISO Dienste hingegen in Programmen wie Citizens, INCO, Research Infrastructures, NEST und Policy, was jedoch wohl in erster Linie auf die Kleinheit der entsprechenden Programme zurückzuführen ist. 
Die PROVISO Produkte sind innerhalb der Zielgruppe von PROVISO weitestgehend gut bekannt, wie aus der Befragung von insgesamt 14 Programmdelegierten bzw. ExpertInnen, 22 BeraterInnen des Bereichs Europäische und Internationale Programme der FFG bzw. der regionalen Beratungs- und Betreuungszentren (RBBZ) sowie 27 externen NutzerInnen von PROVISO hervorgeht (siehe Grafik 3).

Grafik 3 Kenntnis der PROVISO Produkte, nach Zielgruppe, Anteil jener, die das Produkt kennen, in Prozent

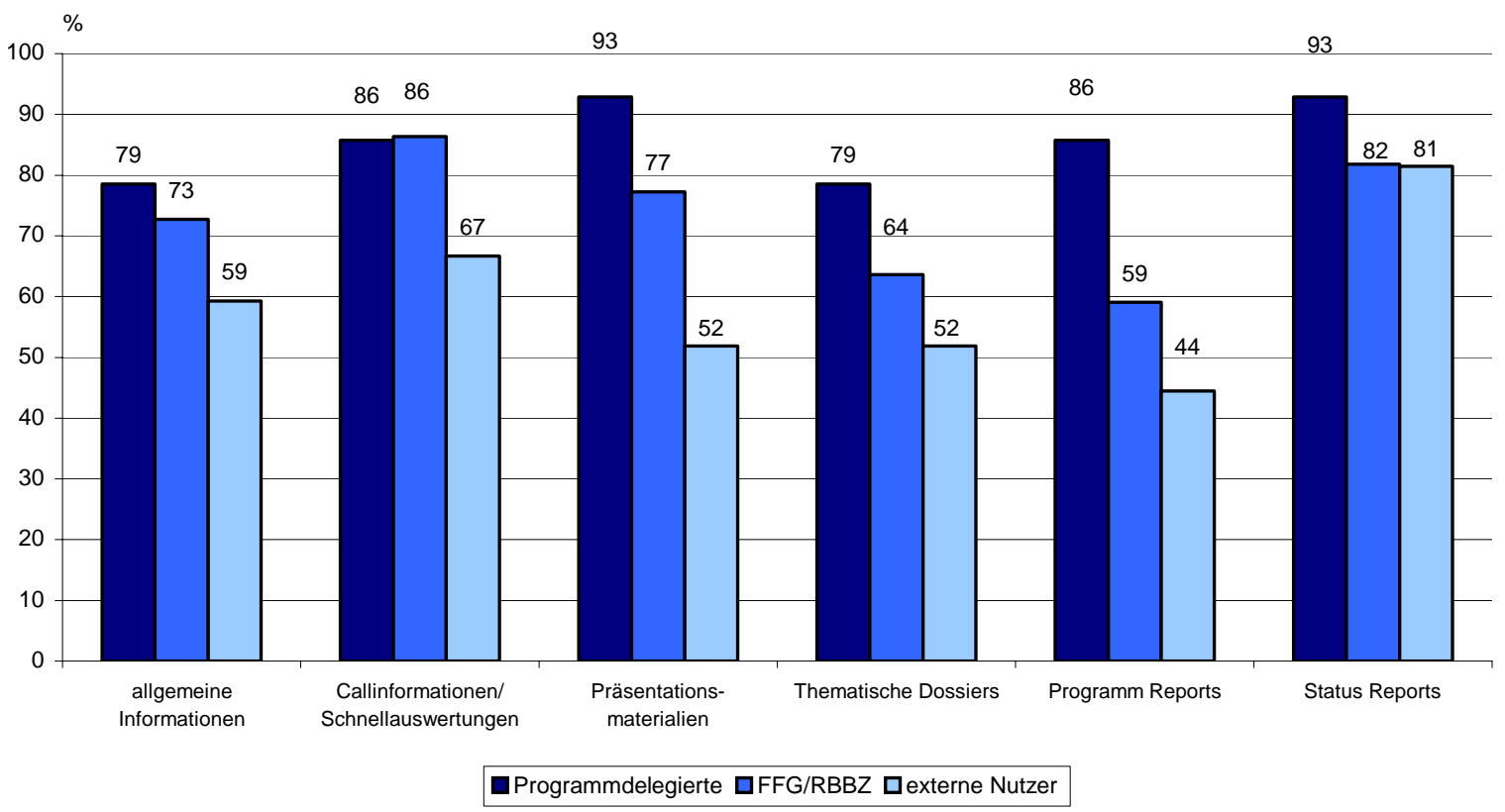

Quelle: KMU FORSCHUNG AUSTRIA, N (PD/E) $=14, \mathrm{~N}(\mathrm{FFG} / \mathrm{RBBZ})=22, \mathrm{~N}$ (externe Nutzer) $=27$

Erwartungsgemäß ist die Kenntnis der unterschiedlichen PROVISO Produkte unter den Programmdelegierten am vergleichsweise höchsten, wenngleich auch mehr als zwei Drittel der FFG/RBBZ-BeraterInnen sowie immerhin über $50 \%$ der externen Nutzer die Produktpalette von PROVISO weitestgehend bekannt ist. Interessant scheint in diesem Zusammenhang jedoch, dass immerhin etwa $15 \%-20 \%$ der Programmdelegierten - als eine der vorrangigen Zielgruppe von PROVISO - in der Befragung angeben, einige der PROVISO Produkte (wie etwa die Möglichkeit, allgemeine Auskünfte/Informationen beziehen zu können, Callinformation/ Schnellauswertungen oder Programm Reports) nicht zu kennen. Hier findet sich möglicherweise ein weiterer Hinweis auf die Notwendigkeit einer klareren Kommunikation der PROVISO Aufgaben und Aktivitäten gegenüber den Programmdelegierten (siehe auch Kapitel 2.1).

\subsection{Nutzung der Produkte und Dienstleistungen von PROVISO}

Die gute Kenntnis der PROVISO Produkte geht größtenteils auch mit einer vergleichsweise hohen Nutzung derselben durch die Zielgruppen von PROVISO einher (siehe Grafik 4). Es wird dabei deutlich, dass die BeraterInnen der FFG bzw. der RBBZ die Produkte genauso intensiv bzw. zum Teil sogar intensiver (z.B. die Callauswertungen/Schnellinformationen, Status Reports und Jahres Reports) nutzen, als die Programmdelegierten. Die im Jänner 2005 erfolgte Ausweitung der Zielgruppe von PROVISO auf die BeraterInnen des Bereichs Europäische und Internationale Programme der FFG sowie auf die regionalen Beratungs- und Betreuungszentren hat somit auch einen wichtigen Beitrag zur effektiveren Nutzung der PROVISO Produkte und Dienstleistungen geleistet, welche gemäß der persönlichen Interviews ein wichtiges Instrument für die Beratungstätigkeit der FFG bzw. RBBZ darstellen. 
Grafik $4 \quad$ Nutzung der PROVISO Produkte, nach Zielgruppe, Anteil jener, die das Produkt nutzen in Prozent

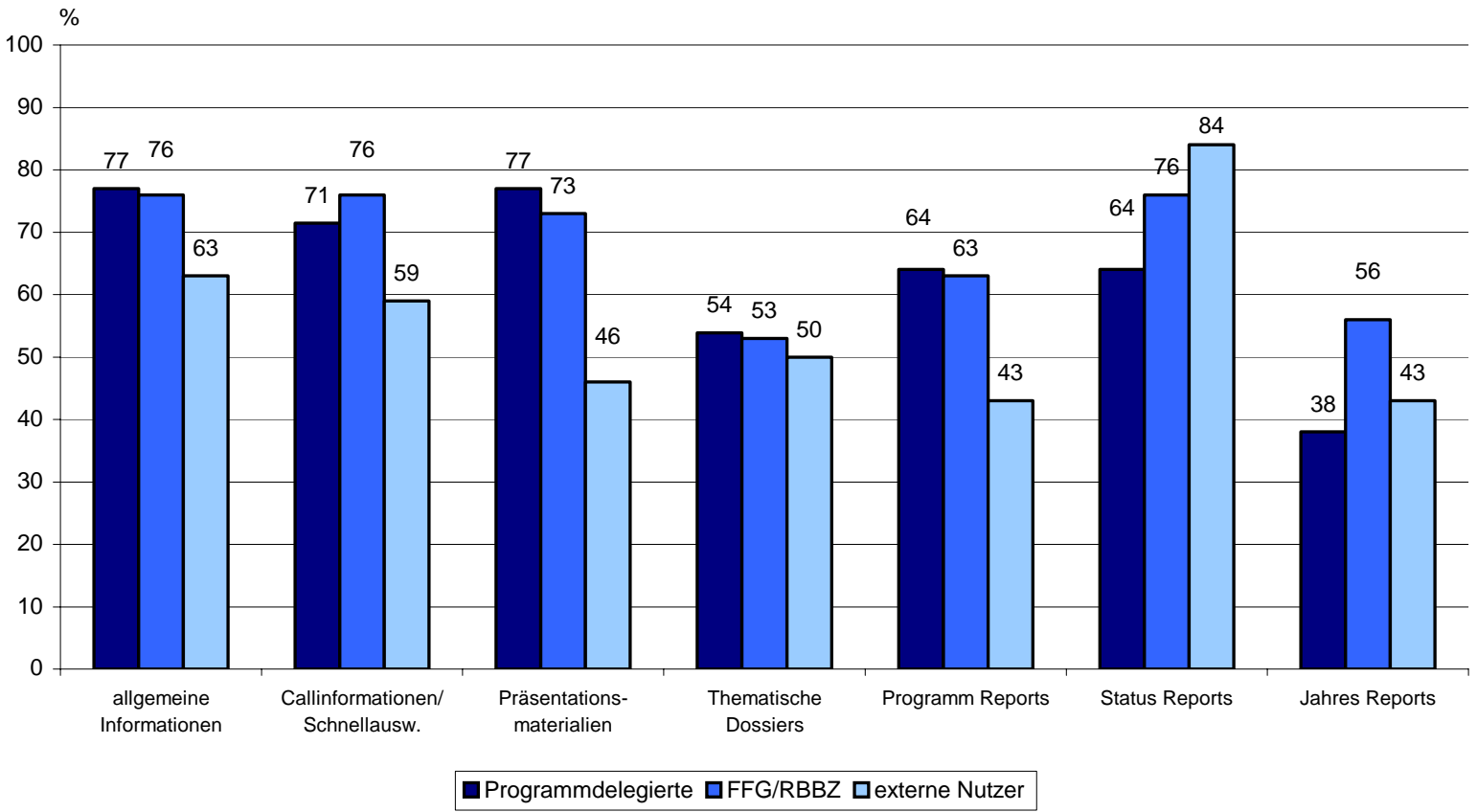

Quelle: KMU FORSCHUNG AUSTRIA, N (PD/E) = 14, N (FFG/RBBZ) = 22, N (externe Nutzer) $=27$

Die Befragung zeigt jedoch auch, dass einige Produkte, wie beispielsweise die Thematischen Dossiers, von knapp der Hälfte der Zielgruppe von PROVISO gar nicht genutzt werden. Auch die Nutzung der Jahres Reports scheint vergleichsweise gering. Da die Relevanz dieser Produkte - vor allem der Thematischen Dossiers - jedoch insbesondere von den Beraterlnnen der FFG/ RBBZ vergleichsweise hoch eingeschätzt wird (siehe auch Grafik 6), wäre hier nach entsprechenden Optimierungsmöglichkeiten zu suchen, um eine effektivere Nutzung bzw. Verwendbarkeit der Produkte zu sicherzustellen.

Die Tatsache, dass immerhin knapp ein Viertel der Programmdelegierten bzw. ExpertInnen einzelne Produkte gar nicht nutzt ist wohl zum einen darauf zurück zu führen, dass diese die Produkte zum Teil nicht kennen (siehe auch oben), zum anderen erhalten einige Programmdelegierte die benötigten Daten auch in vergleichsweise guter Qualität von der EU Kommission. Generell ist jedoch darauf hinzuweisen, dass die Programmdelegierten im Durchschnitt 4 der 7 unterschiedlichen Produkte verwenden, das Spektrum reicht dabei von der Nutzung nur eines Produktes bis zur Verwendung aller 7 Produkte und Dienstleistungen von PROVISO durch die Programmdelegierten und ExpertInnen.

Die PROVISO Produkte werden dabei insgesamt nahezu gleichermaßen auf programmübergreifender wie auf programmspezifischer Ebene verwendet. Während sich die von den Programmdelegierten und ExpertInnen genutzten PROVISO Produkte dabei in erster Linie auf einzelne Programme beziehen, verwenden vor allem die EU-Koordinationsabteilung sowie die externen Nutzer eher programmübergreifende Produkte und Informationen. Nahezu gleichermaßen auf programmübergreifender wie auf programmspezifischer Ebene werden die PROVISO Produkte von den BeraterInnen der FFG sowie der RBBZ genutzt.

Insgesamt finden die PROVISO Produkte und Dienstleistungen überwiegend im nationalen Kontext Verwendung (siehe Grafik 5). Nur insgesamt $8 \%$ der Nutzerinnen verwenden PROVISO ausschließlich im internationalen Kontext, immerhin $40 \%$ nutzen PROVISO gleichermaßen in Zusammenhang mit nationalen sowie internationalen Aktivitäten, dabei ist die Nutzung auf internationaler Ebene bei den Programmdelegierten und ExpertInnen noch am vergleichsweise höchsten unter den verschiedenen Zielgruppen von PROVISO. 


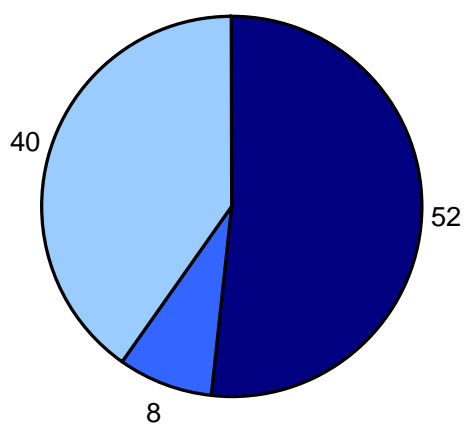

Nutzung eher im nationalen Kontext

口Nutzung eher im internationalen Kontext

口gleichwertige Nutzung international und national

Quelle: KMU FORSCHUNG AUSTRIA, N = 63

Die von PROVISO zur Verfügung gestellten Produkte und Informationen werden von der Zielgruppe von PROVISO im Durchschnitt durchwegs als relevant bzw. sogar sehr relevant beurteilt (siehe Grafik 6). Interessant ist dabei, dass die Relevanz der PROVISO Produkte für ihre Arbeit von den BeraterInnen des Bereichs Europäische und Internationale Programme der FFG bzw. der regionalen Beratungs- und Betreuungszentren - mit Ausnahme der Callinformation/ Schnellauswertungen sowie der Präsentationsmaterialien - höher eingeschätzt wird, als von den Programmdelegierten. Dies trifft insbesondere für die Thematischen Dossiers sowie die Status Reports zu, aber auch die anderen PROVISO Produkte scheinen für die FFG/RBBZ relevanter zu sein, als für die Programmdelegierten. Ähnliches gilt auch für die externen Nutzer von PROVISO, die die Relevanz der PROVISO Produkte für ihre Arbeit - mit den beiden gleichen Ausnahmen wie bei den BeraterInnen der FFG/RBBZ - im Durchschnitt höher einschätzen, als die Programmdelegierten.

Grafik 6 Relevanz der PROVISO Produkte, nach Zielgruppe, Mittelwerte (1 = sehr relevant, 2 = relevant, 3 = weniger relevant, 4 = nicht relevant)

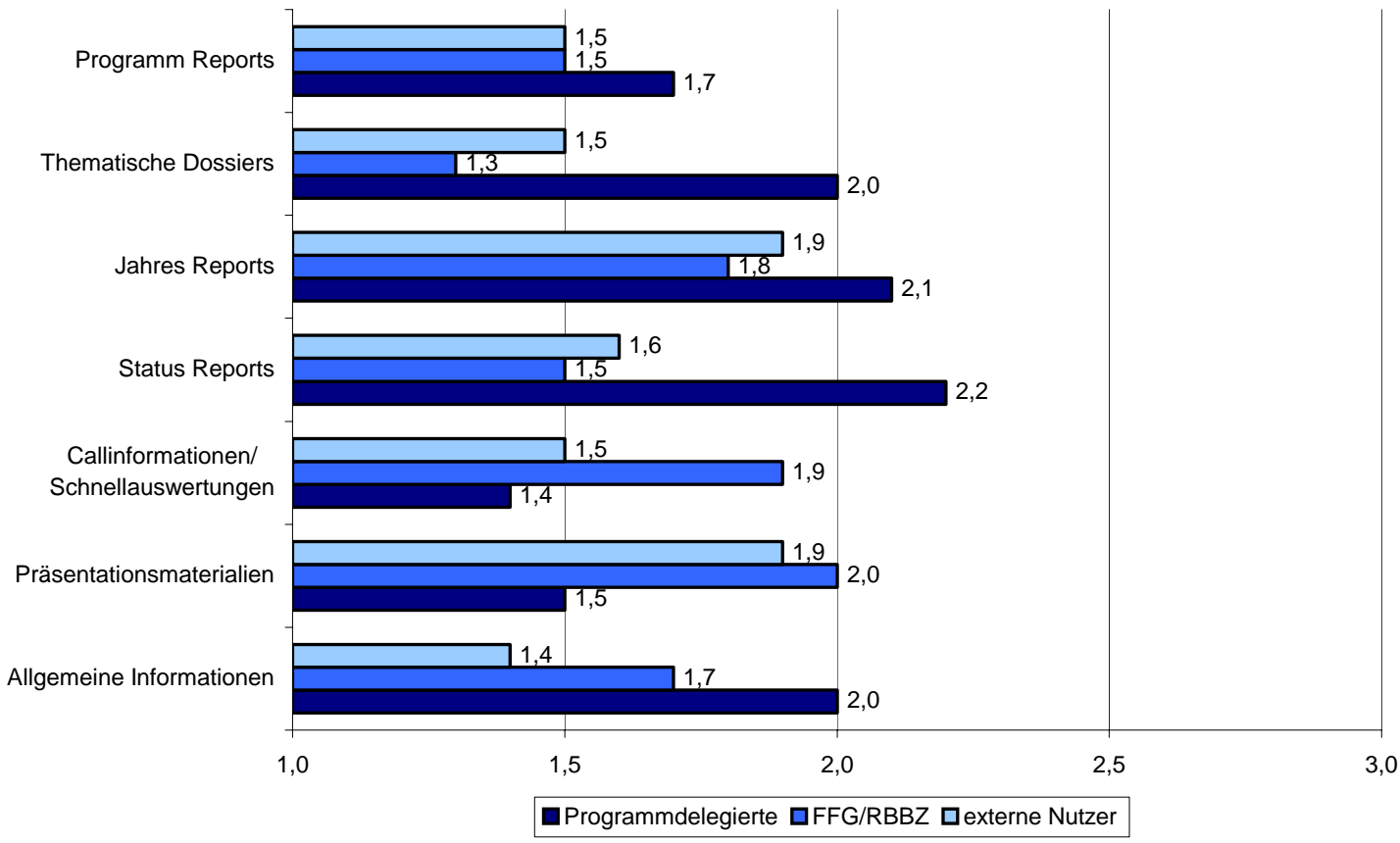

Quelle: KMU FORSCHUNG AUSTRIA, N (PD/E) = 10, N (FFG/RBBZ) = 15, N (externe Nutzer) $=13$ 
Die höhere Einschätzung der Relevanz der PROVISO Produkte für die Arbeit der FFG/RBBZ BeraterInnen im Vergleich zu den Programmdelegierten spiegelt sich auch in der Beurteilung der Bedeutung der PROVISO Produkte und Informationen für die unterschiedlichen Aktivitäten, die von beiden Zielgruppen wahrgenommen werden, wider (siehe Grafik 7). Während PROVISO beispielsweise als Grundlage für die Durchführung von Beratungsgesprächen sowie für die strategische Politikformulierung, insbesondere die Umsetzung der Barcelona-Ziele im Rahmen des Lissabon-Prozesses für die Programmdelegierten weniger wichtig ist, wird es von den FFG/RBBZ-BeraterInnen in Hinblick auf diese beiden Bereiche als durchaus bedeutsam bezeichnet. Auch als Grundlage für ein umfassendes Technologie- und Forschungsmonitoring wird PROVISO von den FFG/RBBZ-BeraterInnen eine höhere Bedeutung beigemessen, als von den Programmdelegierten.

Grafik 7 Bedeutung der PROVISO Produkte und Informationen für unterschiedliche Aktivitäten, nach Zielgruppe*), Mittelwerte (1 = sehr wichtig, 2 = wichtig, 3 = weniger wichtig, $4=$ unwichtig)

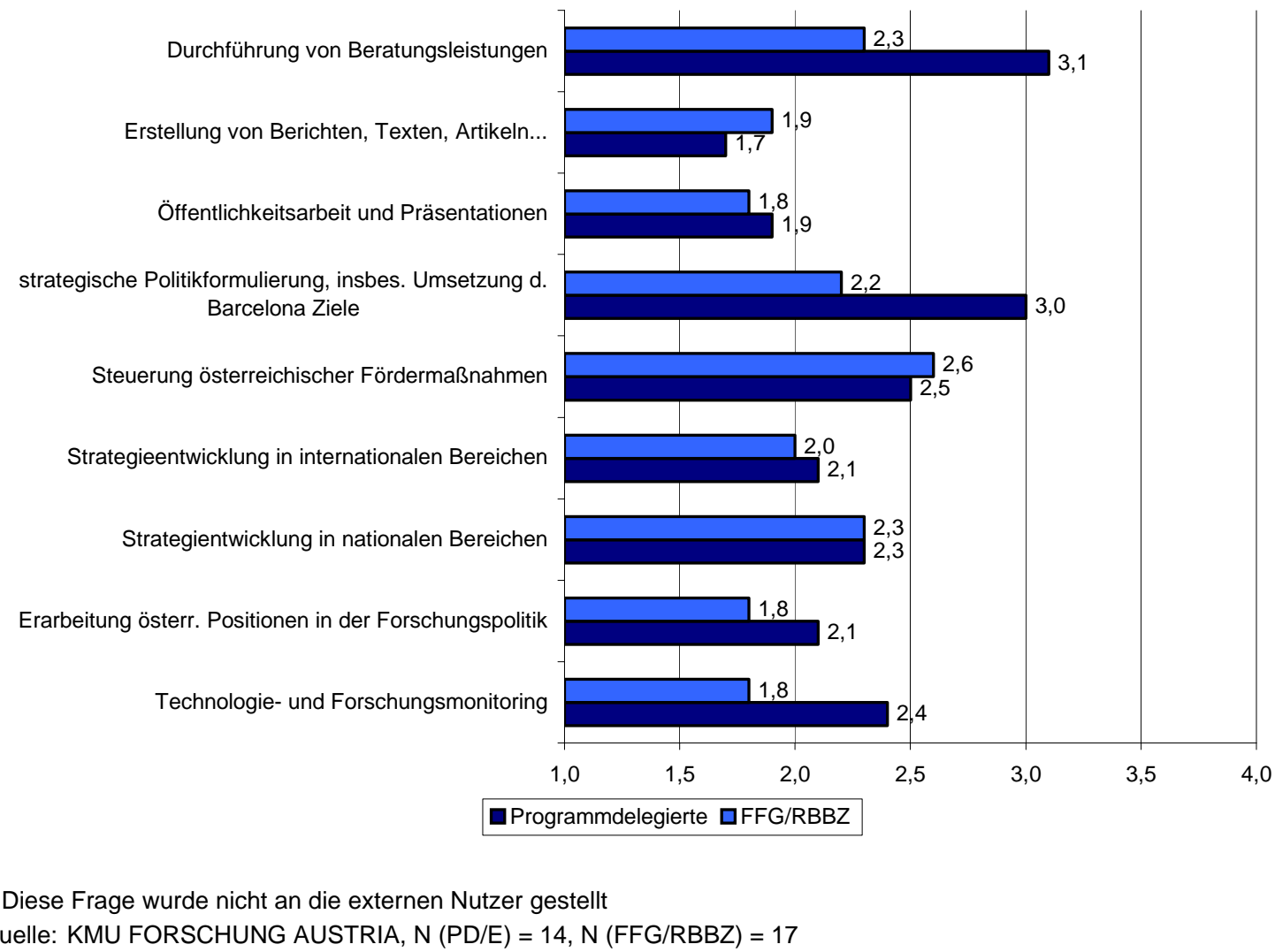

Eine nahezu gleichermaßen hohe Bedeutung kommt hingegen sowohl für die Programmdelegierten und ExpertInnen als auch für die BeraterInnen der FFG/RBBZ den PROVISO Informationen PROVISO als Grundlage für die Erstellung von Berichten, Texten, Artikeln, Informationsbroschüren etc. sowie für die Öffentlichkeitsarbeit und Präsentationen, z.B. bei Informationsveranstaltungen) zu. Ebenfalls wichtig erscheint PROVISO beiden Zielgruppen in Hinblick auf die Strategieentwicklung in internationalen Bereichen sowie die Erarbeitung von österreichischen Positionen in Angelegenheiten der europäischen Forschungs- und Technologiepolitik, wobei die Bedeutung für letzteres von den FFG/RBBZ-BeraterInnen wieder höher eingeschätzt wird als von den Programmdelegierten und ExpertInnen. 


\subsection{Zugang zu den Produkten und Dienstleistungen von PROVISO}

Um auf PROVISO Informationen zuzugreifen, können eine Reihe von Verbreitungskanälen genutzt werden. Als erstes besteht die Möglichkeit, sich (per E-Mail oder Telefon) direkt an PROVISO zu wenden. Sofern die Anfrage durch bestehende Verträge gedeckt ist, erstellen die PROVISO Mitarbeiter/innen nach Rücksprache mit der Projektleitung die Auswertungen und versenden die Ergebnisse im Anschluss per E-Mail. Anfragen seitens der FFG/RBBZ-BeraterInnen oder externer Nutzer, die nicht durch Verträge und allgemeine Zusagen abgedeckt sind (z.B. bei Spezialauswertungen, die vertrauliche Daten beinhalten) bedürfen der Zustimmung des bzw. der zuständigen Programmdelegierten. Die Programmdelegierten erhalten dadurch die Möglichkeit, den Datenfluss gemäß den mit der EU Kommission bestehenden Vertraulichkeitsvereinbarungen besser kontrollieren zu können (siehe auch Kapitel 2.3).

So fern es sich um Standardprodukte handelt, die keine vertraulichen Informationen beinhalten und für die Öffentlichkeit publiziert werden (z.B. Jahres Reports, Programm Reports etc.), können PROVISO Nutzer/innen die Informationen auch von era.gv.at, der elektronischen Projektmanagementplattform EPMP oder von der Homepage von PROVISO downloaden. Bei era.gv.at handelt es sich um ein organisationsübergreifendes Extranet, das sich speziell an Personen richtet, die Interesse an Informationen rd. um EU Forschungsprogramme haben. Eine Registrierung bzw. Anmeldung zu diesem Dienst ist jedoch erforderlich. EPMP ist ein Intranettool, das speziell für die Abwicklung ministeriumsinterner Projekte eingerichtet wurde und auch von PROVISO genutzt werden kann. Der mögliche Nutzerkreis (Ministeriumsmitarbeiter/innen) ist in der Folge gegenüber EPMP kleiner.

Für externe Nutzer am vergleichsweise interessantesten dürfte die Homepage von PROVISO sein, die über http://www.bmbwk.gv.at/proviso erreicht werden kann. $74 \%$ aller PROVISO Nutzer/innen kennen diese Homepage. Dabei ist der Kenntnisstand unter den Programmdelegierten ( $86 \%$ ) deutlich höher als beispielsweise in den Beratungs- und Betreuungseinrichtungen (68\%), während der Kenntnisstand bei den externen NutzerInnen in etwa dem Gesamtdurchschnitt entspricht. Es zeigt sich jedoch, dass die PROVISO Homepage relativ selten genutzt wird: Rd. $38 \%$ der PROVISO Nutzerlnnen greifen hin und wieder, etwa die Hälfte ganz selten und $10 \%$ nie auf die Webseite zu (siehe Grafik 8). Lediglich $2 \%$ bezeichnen sich als regelmäßige User/innen. Die vergleichsweise geringe Nutzungsintensität spiegelt allerdings weniger Unzulänglichkeiten der Internetseite wider, als vielmehr die Tatsache, dass der persönliche Kontakt zu PROVISO von den Beteiligten als vergleichsweise wichtig eingeschätzt wird und auch weitgehend problemfrei funktioniert.

Dementsprechend stellt der direkte Bezug von PROVISO Daten auch die wichtigste Form der Informationsübermittlung an die Zielgruppen von PROVISO dar (siehe Grafik 9). Vor allem die Programmdelegierten wenden sich wegen der von ihnen benötigten Informationen nahezu ausschließlich an die einzelnen PROVISO Mitarbeiter/innen, mit einigen wenigen Ausnahmen, die sich die entsprechenden Daten von der Internetplattform era.gv.at herunter laden. Von der Möglichkeit eines direkten Online-Zugangs zu den PROVISO Daten wird seitens der Programmdelegierten nur in Einzelfällen Gebrauch gemacht. 


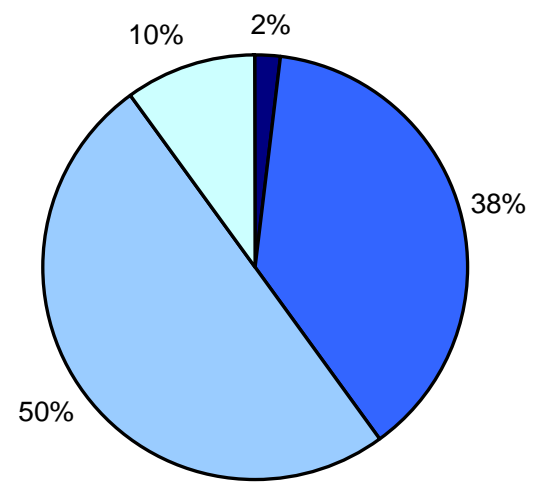

regelmäßig $\square$ hin und wieder $\square$ ganz selten $\square$ nie

Quelle: KMU FORSCHUNG AUSTRIA, N = 50

Der direkte Kontakt zum PROVISO Service ist darüber hinaus auch für die Berater/innen der FFG/RBBZ sowie für die externen Nutzer die wichtigste Bezugsquelle für PROVISO Daten, gefolgt von der PROVISO Homepage und der Internetplattform era.gv.at. Vor allem die externen Nutzer bekommen die von innen benötigten PROVISO Daten und Informationen auch häufig durch Dritte übermittelt, meist durch die Programmdelegierten (über ein Drittel der Programmdelegierten bzw. ExpertInnen gibt die PROVISO Daten regelmäßig und etwas mehr als ein Viertel immerhin hin und wieder an Dritte weiter). EPMP spielt hingegen als Verbreitungskanal für PROVISO keine Rolle.

\section{Grafik 9 Bezugsquellen der PROVISO Produkte, Anteil der Befragten in Prozent}

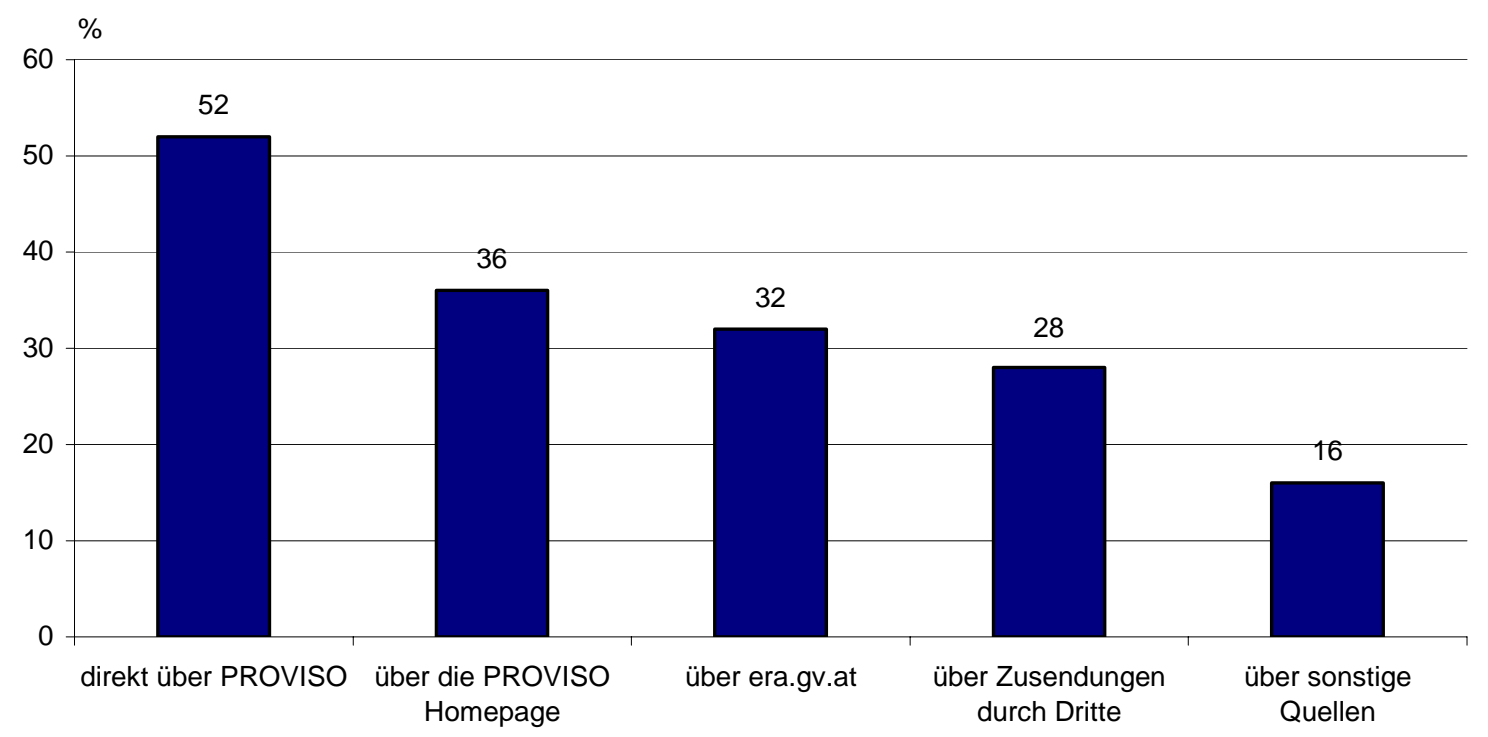

Quelle: KMU FORSCHUNG AUSTRIA, N = 61 
Insgesamt wird der Zugang zu den PROVISO Informationen von den NutzerInnen überwiegend als leicht eingestuft (siehe Grafik 10). Über die Hälfte der Programmdelegierten bzw. immerhin ein Viertel der externen Nutzer von PROVISO empfinden diesen sogar als sehr leicht. Jedoch schätzen auch je ein Fünftel der BeraterInnen der FFG/RBBZ sowie der externen Nutzer, sowie immerhin $8 \%$ der Programmdelegierten den Zugang zu PROVISO eher schwer ein und $11 \%$ der FFG/RBBZ-BeraterInnen sogar als sehr schwer. Dies ist wohl in erster Linie darauf zurückzuführen, dass diese die von ihnen benötigten PROVISO Daten meist nur indirekt über die Programmdelegierten erhalten (siehe oben).

Grafik 10 Bewertung des Zugangs zu PROVISO Produkten, nach Zielgruppe, Anteil in der Zielgruppe in Prozent

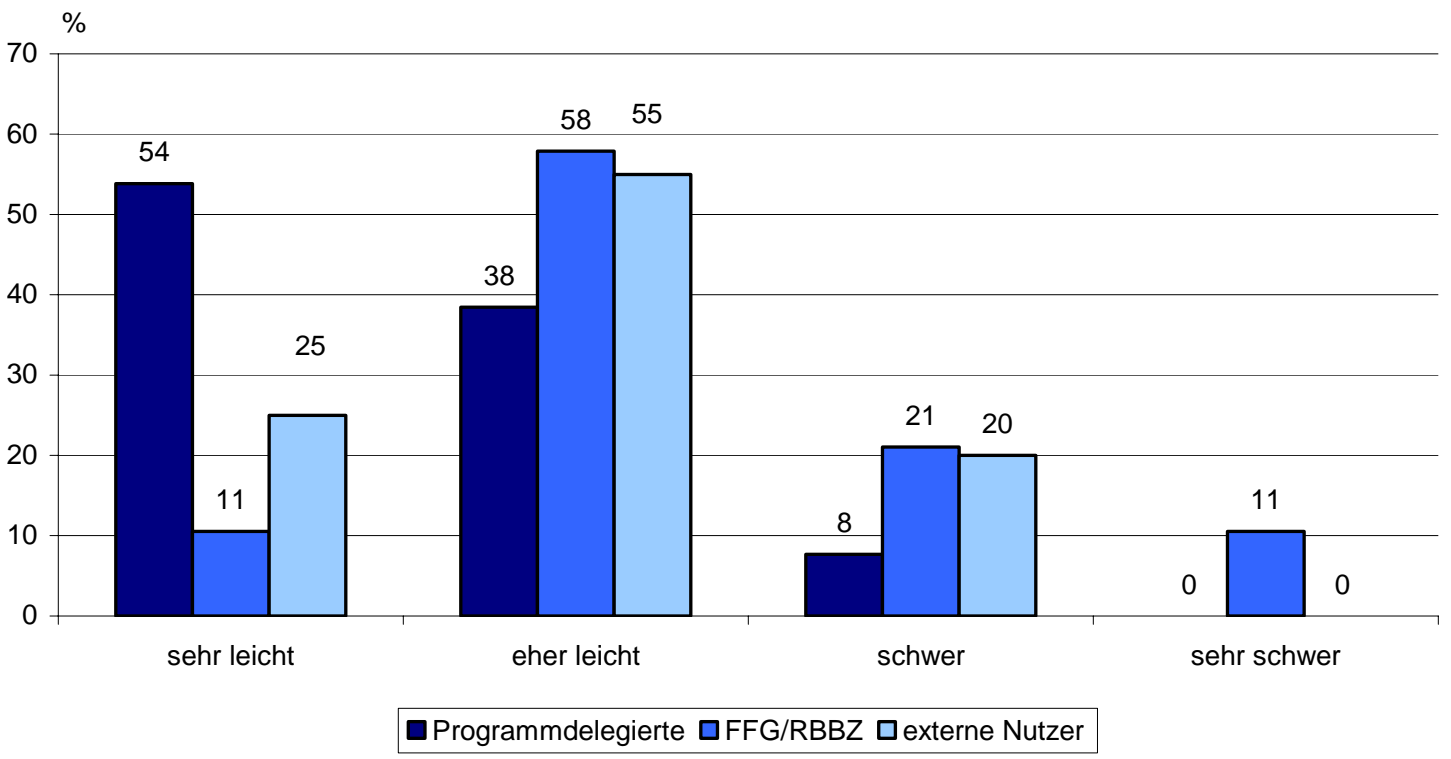

Quelle: KMU FORSCHUNG AUSTRIA, N (PD/E) $=13, N($ FFG/RBBZ) $=19, N($ externe Nutzer $)=20$

Obgleich der Zugang zu PROVISO von den im Rahmen der Fragebogenerhebung befragten externen Nutzern des Service im Durchschnitt als eher leicht eingestuft wird, gibt es Hinweise darauf, dass die Sichtbarkeit von PROVISO in der breiteren Öffentlichkeit bzw. innerhalb der Forschungscommunity nicht sehr hoch ist. Zum einen ist dies mit der derzeitigen Ausrichtung von PROVISO als in erster Linie interne Dienstleistung für die mit den EU-Rahmenprogrammen betrauten Ressorts und Beratungs- und Betreuungsstrukturen zu begründen, zum anderen wurde eine stärkere „Vermarktung" des PROVISO Service - nicht zuletzt auch auf Grund limitierter Kapazitäten innerhalb von PROVISO - bisher seitens der Auftraggeber von PROVISO nicht gefördert. Tatsache ist jedoch, dass sowohl die steigende Anzahl der externen Anfragen an PROVISO (siehe auch Kapitel 2.2) als auch die in Rahmen dieser Evaluierung durchgeführten Interviews darauf hindeuten, dass gerade im Bereich der externen Forschungsgruppen und Organisationen ein zusätzliches Nutzerpotenzial des PROVISO Service besteht. Möglichkeiten, dieses auszuschöpfen müssten einen vertretbaren Aufwand allein deshalb nicht überschreiten, da nach Angaben des PROVISO Service für die Beantwortung externer Anfragen meist auf bestehende PROVISO Produkte zurückgegriffen werden kann. 


\subsection{Zufriedenheit mit dem PROVISO Service}

Generell besteht eine hohe Zufriedenheit mit den PROVISO Produkten und Dienstleistungen seitens der unterschiedlichen Zielgruppen (siehe Grafik 11). Insbesondere die externen Nutzer zeigen sich in allen abgefragten Aspekten der PROVISO Produkte im Durchschnitt weitestgehend zufrieden. Am vergleichsweise schlechtesten wird dabei von allen drei befragten Nutzergruppen der Bereich Klarheit der Erläuterungen bewertet. In diesem Zusammenhang wurde z.B. (sowohl von den Programmdelegierten als auch den Beraterlnnen der FFG/RBBZ) einige Male darauf hingewiesen, dass bei Grafiken zum Teil die zu Grunde liegenden Zahlen nicht mitpubliziert werden oder ein entsprechender Tabellenanhang vielfach fehlt.

Die Übersichtlichkeit der Informationen wird vor allem von den Programmdelegierten am zweit schlechtesten (wenngleich noch immer als zufrieden stellend) bewertet. Die Kritik bezieht sich hier in erster Linie auf: „zum Teil winzig klein geschriebene, riesige Tabellen mit endlos vielen Zahlen drin, die total unlesbar sind“. Zwar hat es hier aus Sicht der Programmdelegierten in der Vergangenheit schon deutliche Verbesserungen gegeben, jedoch besteht nach wie vor zum Teil hohe Unzufriedenheit mit der Darstellung der PROVISO Informationen. Ähnliches gilt in Hinblick auf die sog. Bundesländerauswertungen, die PROVISO den regionalen Beratungs- und Betreuungszentren regelmäßig zur Verfügung stellt, auch hier wird seitens der RBBZ die Überfrachtung und Unübersichtlichkeit der Informationen bemängelt.

Grafik 11 Zufriedenheit mit unterschiedlichen Aspekten der PROVISO Produkte, nach Zielgruppe, Mittelwerte ( 1 = sehr zufrieden, 2 = zufrieden, 3 = weniger zufrieden, 4 = gar nicht zufrieden)

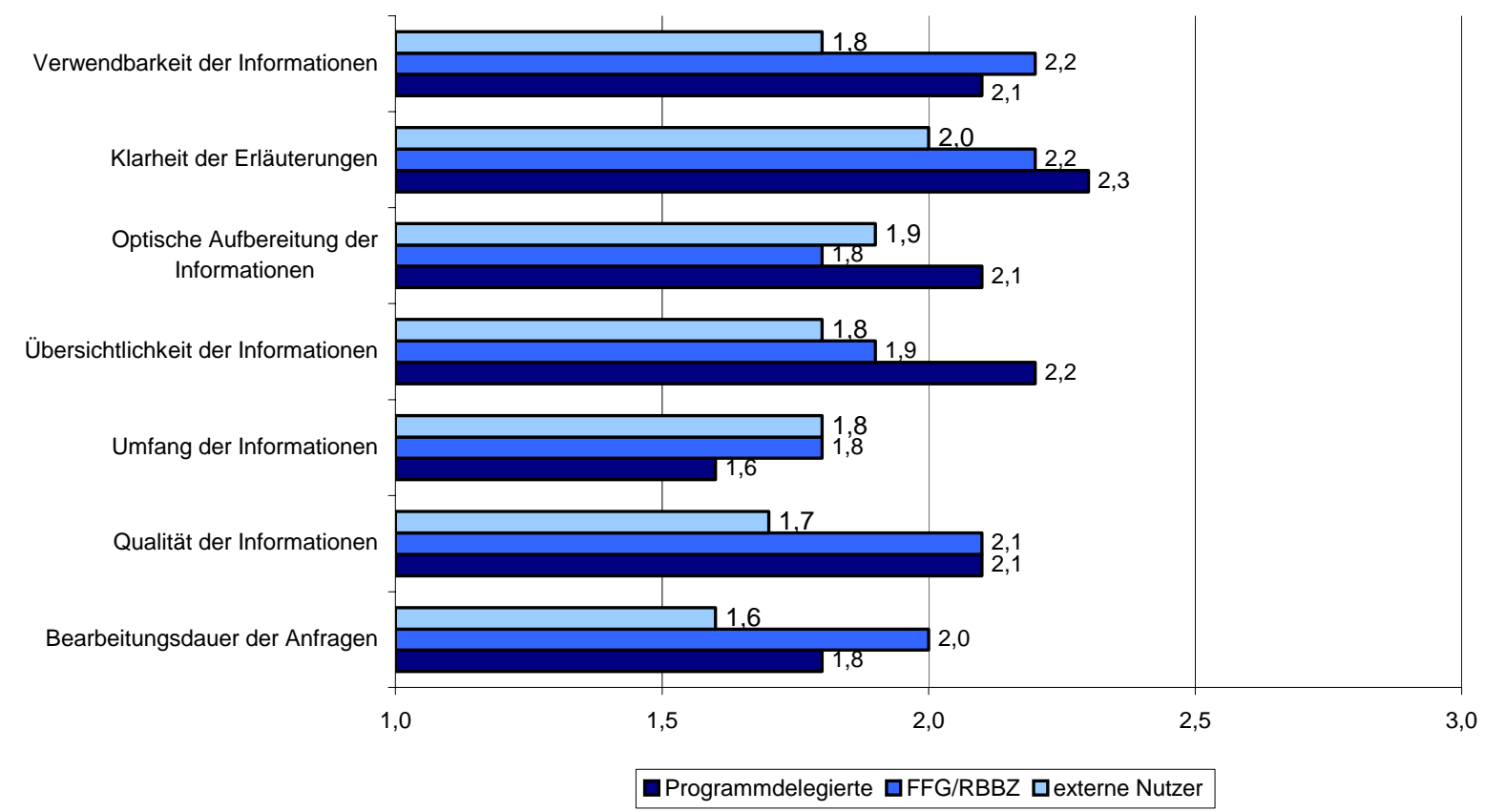

Quelle: KMU FORSCHUNG AUSTRIA, N (PD/E) = 14, N (FFG/RBBZ) = 20, N (externe Nutzer) $=21$

In Bezug auf die Verwendbarkeit der Informationen wird von den Programmdelegierten aber auch von den FFG/RBBZ-BeraterInnen die mangelnde inhaltliche Auseinandersetzung mit den Daten seitens der PROVISO MitarbeiterInnen bzw. die mangelnde Interpretation der zur Verfügung gestellten Daten kritisiert. Insbesondere von einigen Programmdelegierten wird dieser Aspekt als eine deutliche Einschränkung der Verwendbarkeit der Daten empfunden. Vorgeschlagen wurde hier im Rahmen der Interviews unter anderem eine stärkere Einbindung der Programmdelegierten in die Interpretation der Daten. 
Ein weiteres Potenzial in Hinblick auf die Verwendbarkeit der Daten wird vor allem von den Programmdelegierten in der Durchführung detaillierter inhaltlicher Analysen gesehen. Hier wird weitestgehend die Auffassung vertreten, dass PROVISO lediglich Standardauswertungen, jedoch keine tiefer gehenden inhaltlichen bzw. thematischen Analysen produziert (siehe auch Kapitel 2.1). Der Bedarf nach derartigen Analysen scheint jedoch durchaus zu bestehen und ist in der Vergangenheit in Einzelfällen durch externe Beauftragungen gedeckt worden. Die hohe Standardisierung der Produkte bringt des Weiteren mit sich, dass - bedingt durch die gleiche Struktur der Berichte - für einige Programme zum Teil Grafiken produziert werden, die auf Grund einer vergleichsweise geringen Anzahl von Fällen wenig Aussagekraft haben.

Die Qualität der Informationen wird zwar in der Befragung sowohl von den Programmdelegierten als auch von den FFG/RBBZ-BeraterInnen mit einer durchschnittlichen Bewertung von 2,1 als durchaus zufrieden stellend beurteilt, in den persönlichen Interviews wurde jedoch zum Teil deutliche Kritik an der Datenqualität von PROVISO geäußert. Diese bezieht sich in erster Linie darauf, dass den PROVISO MitarbeiterInnen laut der Programmdelegierten auf Grund des gesteigerten Aufwands bei der Datenaufbereitung (siehe Kapitel 2.3) oftmals die Zeit für eine detaillierte inhaltliche Auseinandersetzung mit den Daten fehlt. Zum Teil haben die Programmdelegierten in der Vergangenheit gemäß eigener Angaben - über Ausnahmefälle hinausgehend - fehlerhafte Informationen bzw. Produkte von PROVISO erhalten, die einen umfangreichen Kontrollaufwand seitens der Programmdelegierten erforderlich machen. Hier zeigt sich der Bedarf nach einer verbesserten Qualitätskontrolle der Daten seitens der PROVISO MitarbeiterInnen.

Die Bearbeitungsdauer der Anfragen wird nicht nur von den externen PROVISO-Nutzern am besten im Vergleich zu den anderen Aspekten eingeschätzt, sondern erhält auch von den Programmdelegierten mit 1,8 eine vergleichsweise gute (die zweitbeste) Beurteilung. Am vergleichsweise unzufriedensten mit der Bearbeitungsdauer sind die Beraterlnnen der FFG bzw. der regionalen Beratungs- und Betreuungseinrichtungen. Dies ist wohl in erster Linie darauf zurück zu führen, dass diese ihre Anfragen - auf Grund der Datenschutzproblematik (siehe oben) - häufig erst an die Programmdelegierten richten müssen, welche diese an das PROVISO Service weiter leiten, PROVISO übermittelt die entsprechenden Auswertungen dann an die Programmdelegierten und diese geben sie an die FFG bzW. RBBZ weiter. Dadurch kommt es natürlich zu einer Verlängerung der Bearbeitungsdauer der Anfragen seitens der FFG/RBBZBeraterInnen. Zu prüfen wäre, in wie weit hier nicht ein direkter Zugriff der FFG bzw. der regionalen Beratungs- und Betreuungszentren auf das PROVISO Service möglich ist.

Auch die Zufriedenheit mit dem PROVISO Service selbst ist unter allen NutzerInnen vergleichsweise hoch (siehe Grafik 12). Vor allem die Kompetenz der PROVISO Mitarbeiterlnnen sowie ihre Bereitschaft zur Berücksichtigung individueller Kundenwünsche werden von allen Zielgruppen als sehr gut beurteilt. Aber auch mit der Erreichbarkeit der PROVISO-MitarbeiterInnen sind sowohl die Programmdelegierten und ExpertInnen als die FFG/RBBZ-Beraterlnnen und die externen Nutzern mit jeweils einer durchschnittlichen Bewertung von 1,6 mehr als zufrieden. 
Grafik 12 Zufriedenheit mit dem PROVISO Service, Mittelwerte (1 = sehr zufrieden, 2 = zufrieden, 3 = weniger zufrieden, 4 = gar nicht zufrieden)

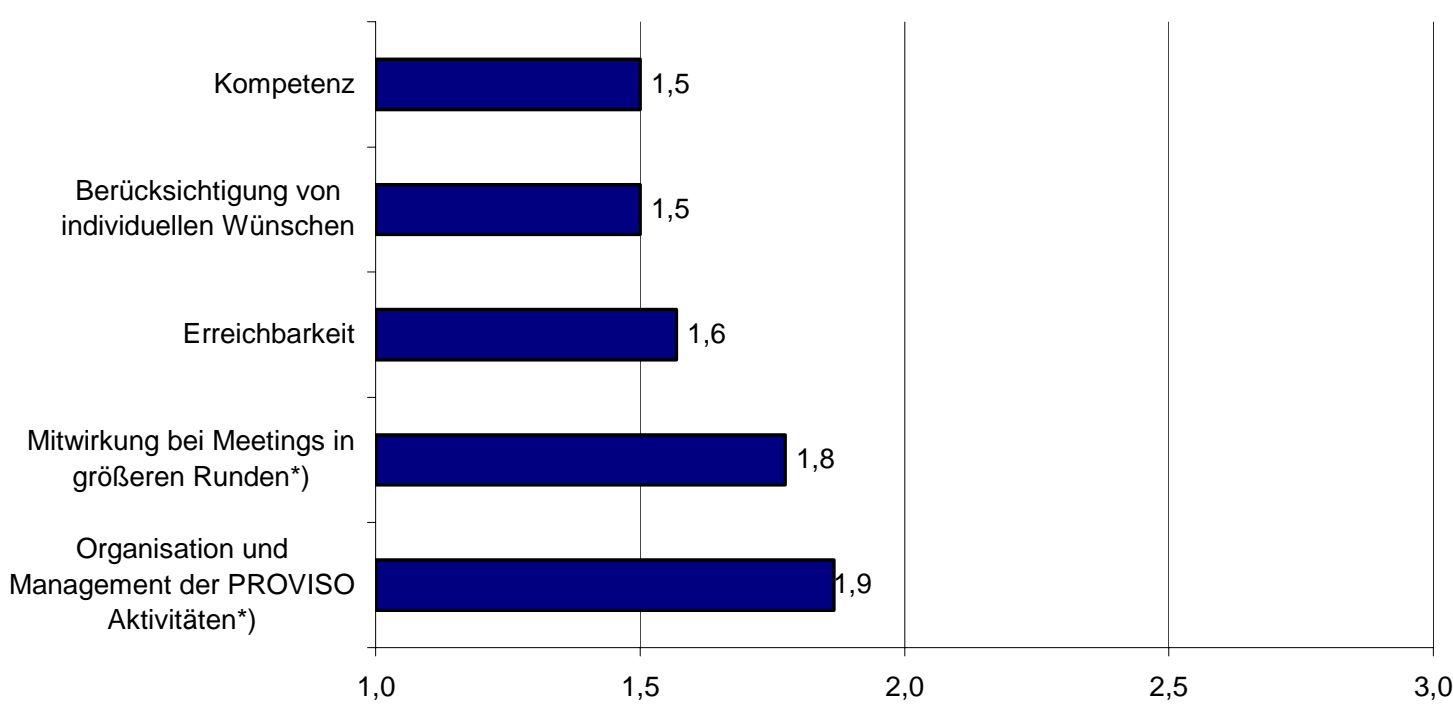

*) wurden nur von den Programmdelegierten bzw. ExpertInnen und den BeraterInnen der FFG/RBBZ bewertet. Quelle: KMU FORSCHUNG AUSTRIA, N (PD/E und FFG/RBBZ) $=33, \mathrm{~N}$ (externe Nutzer) $=18$ 


\section{Analyse der Organisationsstruktur von PROVISO}

\subsection{Organisatorische Einbettung von PROVISO}

In der derzeit bei PROVISO IV genutzten Organisationsform stellt sich PROVISO als relativ eigenständige Serviceeinrichtung innerhalb des BMBWK dar, deren MitarbeiterInnen - einer Stabsstelle ähnlich - ausschließlich Informationen zur österreichischen Beteiligung an den EU Rahmenprogrammen aufbereiten und den Zielgruppen von PROVISO zur Verfügung stellen. Anders als eine "normale" Stabsstelle wird PROVISO jedoch extern abgewickelt: Formal sind die PROVISO MitarbeiterInnen Beschäftigte der Österreichischen Computer Gesellschaft $(\mathrm{OCG})$, die diesbezüglich mit dem BMBWK einen mehrjährigen Rahmenvertrag hinsichtlich des Umfangs, der internen Organisation und der Entlohnung der PROVISO Tätigkeiten abgeschlossen hat. In der Praxis beschränken sich die Tätigkeiten der PROVISO MitarbeiterInnen auf die im Rahmenvertrag zugesicherten PROVISO Leistungen für das Ministerium und weisen darüber hinaus keine Berührungspunkte mit sonstigen OCG Aktivitäten auf. Sämtliche Büros und Arbeitsplätze von PROVISO befinden sich in den Räumlichkeiten des BMBWK.

Die OCG Konstruktion zieht eine Reihe von Konsequenzen nach sich, die allerdings weniger das Tätigkeitsprofil der MitarbeiterInnen betreffen. Tatsächlich dürfte die Abwicklung über die OCG - wie aus zahlreichen Interviews hervorgeht - mit keinen nennenswerten Einschränkungen in Hinblick auf die PROVISO Aktivitäten verbunden sein. Die Kommunikation mit den Programmdelegierten erfolgt meist auf persönlichem, bilateralem Weg und ist unabhängig davon zu sehen, ob die OCG oder das Ministerium selbst für die Gehälter der PROVISO Mitarbeiter/innen aufkommt, das nicht vorhandene formale Weisungsrecht seitens des Ministeriums dürfte in der Praxis ebenfalls keine Rolle spielen. Als Vorteil ist zu werten, dass vielfach kleinere Anschaffungen oder Anträge über die OCG leichter abgewickelt werden können, da die für gröBere Organisationen notwendigen Bewilligungsprozeduren nicht angewendet werden müssen.

Etwas kritischer sind jedoch die unterschiedlichen arbeits- und sozialrechtlichen Regelungen anzusehen. Diese äußern sich sowohl in einer Reihe kleinerer Details, wie beispielsweise der unterschiedlichen (bei PROVISO MitarbeiterInnen doppelt zu erfolgenden) Handhabung der Meldung von Krankständen und Urlauben, aber auch unterschiedlichen Gehaltsschemen, die zuweilen auch Reibungsflächen zwischen den Beschäftigten des BMBWK und PROVISO MitarbeiterInnen hervorrufen können. PROVISO MitarbeiterInnen sind in der Praxis weder typische Angestellte der OCG (da sie mit dieser nur formal verbunden sind) noch - und dies trotz deutlicher Bemühungen um eine weitestgehende Integration - vollwertige Angehörige des BMBWK. Das möglicherweise daraus entstehende Unsicherheitsgefühl könnte bei den PROVISO MitarbeiterInnen mitverantwortlich für die seitens der Programmdelegierten mehrfach erwähnte und kritisierte Personalfluktuation sein (so haben - bei Nichtberücksichtigung von Karenzvertretungen - seit Ende Juli 20045 MitarbeiterInnen PROVISO verlassen und wurden durch anderen ersetzt).

Die - unabhängig von der OCG Lösung zu sehende - Einbettung von PROVISO in die Ministerialstruktur bringt eine Reihe von Vorteilen. So ermöglicht die „in-house“ Verfügbarkeit vor allem den Programmdelegierten und der EU-Koordinationsabteilung einen raschen Zugriff auf die benötigten Informationen, die interne Abstimmung und Koordination wird erleichtert und der vertrauliche Umgang mit den Daten der EU Kommission ist vergleichsweise unproblematisch zu handhaben. Andererseits dürften Synergiepotenziale zwischen PROVISO und den Beratungs- und Betreuungseinrichtungen, die sich aus dem Beratungs-Know-How sowie der insbesondere innerhalb der FFG bereits bestehenden Infrastruktur ergeben weitestgehend ungenutzt bleiben. 


\subsection{Interne Organisation der PROVISO Aktivitäten}

\section{Personalstruktur und interner organisatorischer Aufbau}

PROVISO weist, bedingt auch durch die Größe der Serviceeinrichtung, eine sehr flache Organisationsstruktur auf. Das Projektteam besteht aus 7 Personen mit regulären Dienstverhältnissen, wobei eine Person die Projektleitung und damit die Hauptverantwortung innehat (siehe Tabelle 2). Das Beschäftigungsausmaß beträgt bei 4 der PROVISO MitarbeiterInnen 38,5 Stunden die Woche, bei den übrigen 20 Stunden die Woche. Dies entspricht insgesamt 5,5 Vollzeitäquivalenten. Die Verschlechterung der Qualität der von der EU-Kommission gelieferten Daten sowie die strukturellen Änderungen in der Programmstruktur des 6. EU-Rahmenprogramms (siehe Kapitel 2.3) bedingen im Vergleich zu PROVISO I bis III einen höheren Bedarf im Bereich der Datenaufbereitung und -administration, der $u$. a. durch zusätzliche externe Kräfte auf Werkvertragsbasis abgedeckt wird. September 2005 waren zwei WerkvertragsstudentInnen für PROVISO tätig.

Die Arbeitsteilung zwischen den PROVISO Mitarbeiter/innen erfolgt hauptsächlich entlang der Programme des 6. EU Rahmenprogramms bzw. deren inhaltlicher Verwandtheit. Damit folgt PROVISO intern auch den inhärent vorgegebenen Zuständigkeitsfeldern der Programmdelegierten, was bewirkt, dass jede/r PROVISO Mitarbeiter/in bestimmten Programmdelegierten zugeordnet ist. Bedingt durch die Heterogenität der verschiedenen Programme im 6. EU Rahmenprogramm, aber auch auf Grund unterschiedlicher Präferenzen der Programmdelegierten in Hinblick auf die Art und Darstellungsform von Auswertungen, unterscheiden sich die Tätigkeiten der einzelnen PROVISO Mitarbeiter/innen dabei in einer Vielzahl von Detailaspekten. Daraus lässt sich auch ein relativ hoher Einarbeitungs- und Schulungsaufwand ableiten, der die Substituierbarkeit der Tätigkeiten von PROVISO Mitarbeiter/innen untereinander empfindlich einschränkt. Insbesondere vor dem Hintergrund der unvermutet schlechten Datenlage dürfte der Einarbeitungsaufwand in der Startphase von PROVISO IV unterschätzt worden sein, was entsprechende Verzögerungen bei der Bearbeitung von Anfragen verursachte.

Die Ausbildungshintergründe der PROVISO Mitarbeiter/innen (durchwegs Akademikerlnnen) weisen großteils einen inhaltlichen Bezug zu den betreuten Programmen auf. So ist z. B: eine Genetikerin für das Programm LIFESC, eine Physikerin für das Programm ENERGY und eine Ökologin für das Programm FOOD zuständig. Für die Rekrutierung von Personal gibt es intern Stellenbeschreibungen. 
Tabelle 2 Das PROVISO Projektteam

\begin{tabular}{|c|c|c|c|}
\hline Person & $\begin{array}{l}\text { Ausbildungs- } \\
\text { hintergrund }\end{array}$ & Programmzuständigkeit & $\begin{array}{c}\text { Beschäftigungs- } \\
\text { ausmaß }\end{array}$ \\
\hline \multicolumn{4}{|l|}{ Projektleitung } \\
\hline $\begin{array}{l}\text { Mag. MA Michaela } \\
\text { TOPOLNIK } \\
\text { (bis Februar 2005) }\end{array}$ & $\begin{array}{l}\text { Politik- } \\
\text { wissenschaft } \\
\text { Internationale } \\
\text { Ökonomie }\end{array}$ & $\begin{array}{l}\text { - } \quad \text { horizontale Programme: } \\
\text { SSP/NEST, INCO, SME, ERA- } \\
\text { NET, INFRASTRUCTURES }\end{array}$ & 38,5 Std./Woche \\
\hline $\begin{array}{l}\text { Mag. Günter DINHOBL } \\
\text { (seit März 2005) }\end{array}$ & Physik & $\begin{array}{ll}\text { - } & \text { horizontales Programm } \\
\text { ERA-NET }\end{array}$ & 38,5 Std./Woche \\
\hline \multicolumn{4}{|l|}{ Mitarbeiter/innen } \\
\hline Mag. Karin MÜLLER & $\begin{array}{l}\text { Politik- } \\
\text { wissenschaft }\end{array}$ & $\begin{array}{ll}\text { - } & \text { MOBILITY } \\
\text { - } & \text { SCIENCE+ SOCIETY } \\
\text { - } & \text { CITIZENS }\end{array}$ & 38,5 Std./Woche \\
\hline Dr. Harald F. SIGMUND & Biochemie & $\begin{array}{ll}- & \text { IST } \\
- & \text { AERO/SPACE } \\
- & \text { horizontale Programme: SSPI } \\
& \text { NEST, INCO, SME }\end{array}$ & 38,5 Std./Woche \\
\hline $\begin{array}{l}\text { DI Margit EHARDT- } \\
\text { SCHMIEDERER }\end{array}$ & $\begin{array}{l}\text { Landwirtschaft } \\
\text { (BOKU), } \\
\text { Zusatzausbildung } \\
\text { in Mediation }\end{array}$ & $\begin{array}{ll}\text { - } & \text { GLOBAL } \\
\text { - } & \text { NMP } \\
\text { - } & \text { EURATOM } \\
\text { - } & \text { JRC } \\
\text { - } & \text { INFRASTRUCTURES } \\
\end{array}$ & 38,5 Std./Woche \\
\hline Mag. Tamara COJA & Ökologie & - $\quad$ FOOD & 20 Std./Woche \\
\hline Dr. Maria HEINRICH & Genetik & - $\quad$ LSH & 19 Std./Woche \\
\hline Mag. Maria RAMADORI & Physik & $\begin{array}{ll}\text { - } & \text { ENERGY } \\
\text { - } & \text { TRANSPORT } \\
\end{array}$ & 20 Std./Woche \\
\hline
\end{tabular}

Sollte eine Stelle innerhalb von PROVISO neu ausgeschrieben werden, so entscheidet der dieser Stelle zugeordnete Programmdelegierte aus einem Dreiervorschlag der PROVISO Projektleitung (sofern diese Stelle einen direkten Bezug zu einem Programmdelegierten hat, ansonsten übernimmt die Koordinationsabteilung als Auftraggeber diese Aufgabe).

\section{Kommunikationsflüsse in und um PROVISO}

Der Größe des Personalstandes und der direkten Einbettung in die Ministerialstruktur entsprechend wird innerhalb von PROVISO überwiegend informell kommuniziert. An formalen Kommunikationsflüssen existiert lediglich ein zweiwöchig anberaumtes PROVISO internes Meeting, in dem der aktuelle Stand der PROVISO Aktivitäten erörtert wird. Um PROVISO besser in die Ministeriumsabläufe zu integrieren, ist es PROVISO Mitarbeiter/innen zudem freigestellt, an den wöchentlichen Abteilungsmeetings teilzunehmen. Für die PROVISO Projektleitung ist die Teilnahme verpflichtend. 
Nach außen hin kommuniziert PROVISO in erster Linie über die Projektleitung. Sollte ein Programmdelegierter eine Sonderauswertung wünschen, muss die zuständige PROVISO Mitarbeiterln formal dies der PROVISO Projektleitung melden und die entsprechende Zusage abwarten. Diese für das Projektcontrolling durchaus sinnvolle Prozedur dürfte in einigen Fällen und vor dem Hintergrund der Anlaufschwierigkeiten (erhöhte Bearbeitungsdauer auf Grund der schlechten Datenqualität) dazu geführt haben, dass einige NutzerInnen sich mit Anfragen wegen der vermeintlich höheren Bearbeitungszeit zurückgehalten haben. Hier spielt auch hinein, dass Planangaben von PROVISO zu der Anzahl zu erstellender Produkte hinsichtlich ihrer Bedeutung einigen Delegierten vermutlich zu unklar kommuniziert wurden: Als Vorausschau für das nächste Jahr schätzt PROVISO die Anzahl möglicher Anfragen/Produkte am Ende jedes Jahres, auch getrennt nach Zielgruppen/Auftraggeber. Diese Planwerte sind jedoch kein festgelegtes Budget, bis zu welchem Anfragen von einem Nutzer durchgeführt werden können, sondern rein rechnerische Größen zu PROVISO internen Controllingzwecken.

Die - durch die Datenschutzproblematik bedingte - Zwischenschaltung der Programmdelegierten in der Kommunikation von PROVISO mit den Beratungs- und Betreuungseinrichtungen und weitergehenden externen Nutzer/innen ist ein zentrales Organisationsmerkmal von PROVISO. Gemeinsam mit der Tatsache, dass die Delegierten auch die vorrangige Kundengruppe für PROVISO darstellen, kommt der Kommunikation zwischen PROVISO und den Delegierten eine hohe Bedeutung zu. Die regelmäßig stattfindenden Quartalsmeetings bzw. die EFF Vorbereitungsmeetings scheinen den Abstimmungsbedarf zwischen PROVISO und den Programmdelegierten jedoch nicht in ausreichendem Maße abzudecken, vor allem seitens der Programmdelegierten wird hierbei die Notwenigkeit einer intensiveren Zusammenarbeit unter anderem auch auf bilateraler Ebene geäußert, um die Qualität und Nutzbarkeit der von PROVISO zur Verfügung gestellten Daten zu gewährleisten. Insbesondere wird darauf hingewiesen, dass der als zu gering empfundene Diskussionsprozess im Vorfeld der Veröffentlichung von PROVISO Produkten (v.a. von programmübergreifender Auswertungen), in Einzelfällen zu fehlerhaften Schlussfolgerungen innerhalb der PROVISO Produkte führen kann, bzw. in der Vergangenheit geführt hat. Hier wäre eine intensivere - gegebenenfalls auch institutionalisierte - bilaterale Zusammenarbeit zwischen den PROVISO MitarbeiterInnen und den jeweiligen Programmdelegierten an zudenken, um eine Optimierung des Qualitätsniveaus der von PROVISO zur Verfügung gestellten Produkte und Informationen zu erreichen.

\section{Projektmanagement und Controlling}

Das Projektmanagement bzw. das Projektcontrolling nutzt als primäres Tool ein umfangreiches Zeiterfassungssystem. Dieses System erlaubt die Aufschlüsselung aller PROVISO Aktivitäten in Viertelstundenschritten entlang von 84 Standardtätigkeiten (wie Berichtsteile schreiben, Dateneingabe etc.), die in 9 Kategorien unterteilt sind. Alle Standardtätigkeiten können bzw. müssen in einem weiteren Schritt einzelnen Programmen, Auftraggebern und Produkten zugeordnet werden. Daneben sind von den PROVISO Mitarbeiter/innen Stunden- und Anwesenheitslisten für die OCG zu führen. Die hohe Erfassungsdichte ermöglicht detaillierte Auswertungen bezüglich des Zeitaufwandes für die Erstellung von PROVISO Produkten bzw. die Beantwortung von Anfragen.

Hinsichtlich der Qualitätssicherung kommen hauptsächlich einfache, aber der Größe der Serviceeinrichtung entsprechend durchaus adäquate Prozeduren (wie gegenseitiges Korrekturlesen, Plausibilitätstests etc.) zum Einsatz. Bei der Bewertung der Effektivität der PROVISO Qualitätssicherung ist einerseits die PROVISO zu Grunde liegende Datenqualität der von der EU Kommission gelieferten Daten und anderseits der Trade-Off zwischen Aktualität und Richtigkeit der Informationen (z.B. bei den Schnellauswertungen, die naturgemäß in geringerem Ausmaß kontrolliert werden können) zu berücksichtigen. Ansatzpunkte für eine verbesserte Qualitätssicherung stellen hier die weiter oben erwähnte verstärkte Einbindung der Delegierten, aber, so weit möglich, auch der Beratungs- und Betreuungseinrichtungen im Vorfeld der Produkterstellung dar. 


\section{Schlussfolgerungen und Handlungsanregungen}

Insgesamt wird sowohl den Produkten und Dienstleistungen von PROVISO als auch dem PROVISO Service selbst ein sehr gutes Zeugnis von seinen NutzerInnen ausgestellt. Die Relevanz und Bedeutung des Service für die österreichische Forschungspolitik ist unbestritten, dies bestätigt sich auch in den zahlreich geführten Interviews. Österreich scheint hier eine Pionierstellung einzunehmen, andere Länder beginnen erst mit dem Aufbau ähnlicher Systeme (siehe auch Anhang). Verbesserungspotenziale des Service liegen daher eher im Detail bzw. betreffen die zukünftige Positionierung von PROVISO in einem Umfeld, in dem sich die Rahmenbedingungen in den vergangenen Jahren zum Teil deutlich gewandelt haben. Im Folgenden werden daher aus den vorangegangenen Analysen einige Schlussfolgerungen gezogen sowie Handlungsanregungen für zukünftige Entwicklungen aufgezeigt.

\section{Zielsetzung und Aufgaben von PROVISO}

Das Ziel von PROVISO ist die Sicherstellung eines bestmöglichen Monitorings der österreichischen Teilnahme am 6. EU-Rahmenprogramm für Forschung, technologische Entwicklung und Demonstration im internationalen Vergleich, mit der Zielsetzung der Erfolgskontrolle, der Schaffung von Grundlagen für die Steuerung der österreichischen Fördermaßnahmen sowie einer Referenz für die Politikformulierung im Hinblick auf strategische europäische Entscheidungs- und Koordinationsprozesse. Neben dieser „Monitoring-Funktion“ hat PROVISO auch eine sog. „Service-Funktion“, nämlich die Erarbeitung von Auswertungen und Analysen für die Programmdelegierten als Grundlage für ihre steuerungspolitischen Managementaufgaben sowie für die anderen Zielgruppen von PROVISO.

Während die Bedeutung von PROVISO als Monitoring-Instrument außer Frage steht und hier den Anforderungen der Auftraggeber entsprechend von PROVISO, insbesondere für die EUKoordinationsabteilung, eine fundierte Informationsbasis für die Strategieentwicklung (auf nationaler und internationaler Ebene) und die Politikberatung zur Verfügung gestellt wird, sind Ansatzpunkte für Verbesserungspotenziale vor allem im Bereich der „Service-Komponente“ von PROVISO zu sehen. Dies setzt zum einen an einer Optimierung der Qualität der zur Verfügung gestellten Daten an (siehe auch unten), wofür entsprechende Maßnahmen (gegebenenfalls unter Einbeziehung der Programmdelegierten als eine der vorrangigen Zielgruppe von PROVISO) erarbeitet werden sollten. Zum anderen bestehen insbesondere seitens der Programmdelegierten zum Teil sehr unterschiedliche Erwartungen und Anforderungen an die "ServiceFunktion" von PROVISO, denen es durch eine klare und transparente Definition und Kommunikation der Aufgaben und Leistungen von PROVSIO zu begegnen gilt, um eine effektive Nutzung des PROVISO Service zu gewährleisten.

\section{Zielgruppen von PROVISO}

Die mit PROVISO geschaffene Informationsstruktur bildet die Basis für ein fundiertes Technologie- und Forschungsmonitoring der österreichischen Forschungsaktivitäten im europäischen Umfeld, dessen Relevanz insbesondere auf einer programmübergreifenden Ebene von allen befragten Akteuren außer Frage gestellt wird. Damit wird insbesondere den Anforderungen der Auftraggeber an PROVISO Rechnung getragen, denen PROVISO eine wichtige Grundlage für die Strategiebildung und Politikberatung zur Verfügung stellt. 
Auf programmspezifischer Ebene wird der Nutzen von PROVISO hingegen eher heterogen beurteilt und hat für einige Programmdelegierte nach eigenen Angaben mit dem Wegfall der Assistenztätigkeit deutlich abgenommen. Während PROVISO jedoch für viele Programmdelegierte dennoch eine durchaus wichtige Informationsbasis für ihre tägliche Arbeit bildet, wird der Service in anderen Programmen wenig genutzt. Diese Heterogenität spiegelt zum Teil den unterschiedlichen Bedarf an PROVISO Daten (beispielsweise auf Grund einer hohen Qualität der von der EU-Kommission gelieferten Daten innerhalb einzelner Programme) wider, andererseits drückt sich hierin auch die unterschiedliche Zufriedenheit mit der Qualität der von PROVISO gelieferten Daten aus. Hier eine Verbesserung des Service-Niveaus gegebenenfalls zu Lasten der Produkt-Vielfalt zu erreichen, wird eine der wesentlichen Vorraussetzung sein, um in Zukunft den Anforderungen der Programmdelegierten - als eine der vorrangigen Zielgruppe von PROVISO - besser gerecht werden zu können.

Mit der Aufnahme des Bereichs Europäische und Internationale Programme der FFG sowie der regionalen Beratungs- und Betreuungszentren (RBBZ) als Zielgruppe von PROVISO konnte der Kreis der PROVISO Adressaten um eine wichtige Nutzergruppe erweitert und damit die Effektivität der der PROVISO Produkte und Informationen deutlich gesteigert werden. Zum Teil wird die Relevanz der PROVISO Produkte von den BeraterInnen der FFG bzw. der RBBZ für ihre tägliche Arbeit sogar höher eingeschätzt als von den Programmdelegierten. Für die FFG und die RBBZ stellt PROVISO eine wichtige Grundlage in Hinblick auf ihre Beratungstätigkeit dar, Einschränkungen in der Nutzbarkeit der Daten sind jedoch - auf Grund der bestehenden Datenschutzproblematik - vor allem durch die zum Teil deutlich längeren Bearbeitungszeiten sowie eine zum Teil nicht zielgerechte Aufbereitung der Daten durch PROVISO für die Zwecke dieser Zielgruppe gegeben, die unter anderem der Entstehung von Parallelstrukturen in der FFG Vorschub leistet.

Eine zunehmende Bedeutung erlangen auch externe Forschungsgruppen und Organisationen als Nachfrager und Nutzer von PROVISO Leistungen. Derzeit stellen diese keine primäre Zielgruppe von PROVISO dar, die steigende Anzahl externe Anfragen seitens dieser Gruppe sowie die geführten Interviews bestätigen jedoch ein zusätzliches Nutzerpotenzial des PROVISO Service im Bereich externer Forschungsgruppen und Organisationen. In wie weit die Ausschöpfung dieses Potenzials eine zukünftige Herausforderung für PROVISO darstellen könnte, dass ursprünglich als ministeriumsinternes Dienstleistungsinstrument konzipiert wurde, wäre abzuwägen. Tatsache ist jedoch, dass in diesem Bereich eine zunehmende Nachfrage nach PROVISO Leistungen besteht, die, beispielsweise durch eine automatisierte E-MailVersendung bestehender relevanter PROVISO Produkte (basierend auf aggregierten Daten) an entsprechend registrierte Forschungsgruppen und Organisationen auf durchaus effektive Weise befriedigt werden könnte.

\section{Produkte und Dienstleistungen von PROVISO}

Die PROVISO Produkte und Dienstleistungen stellen einen wichtigen Bestandteil für die tägliche Arbeit ihrer Zielgruppen, insbesondere für die Programmdelegierten und BeraterInnen der FFG bzw. der regionalen Beratungs- und Betreuungszentren, dar. Dabei ist die Nutzung der PROVISO Produkte durch die FFG bzw. RBBZ zum Teil sogar noch intensiver als durch die Programmdelegierten. Dennoch unterscheidet sich die Nutzungsintensität zum Teil deutlich zwischen den einzelnen Produkten, während beispielsweise auf allgemeine Informationen, Callauswertungen/Schnellberichte oder Präsentationsmaterialien vergleichsweise häufig zurückgegriffen wird, werden die Thematischen Dossiers oder Jahres Reports von knapp der Hälfte der Zielgruppe von PROVISO gar nicht genutzt. Hier gilt es Möglichkeiten einer Reduktion der Produktvielfalt zugunsten einer Optimierung der Datenqualität auszuloten bzw. entsprechende Optimierungsbereiche ausfindig zu machen, um eine effektivere Nutzung bzw. Verwendbarkeit dieser Produkte sicherzustellen. 
Generell wird den Produkten und Dienstleistungen von PROVISO jedoch eine hohe Zufriedenheit seitens der unterschiedlichen Zielgruppen bescheinigt. Verbesserungspotenziale liegen neben einigen Detailbereichen, die in erster Linie die Darstellung bzw. Übersichtlichkeit der zur Verfügung gestellten Informationen betreffen, vor allem im Bereich der Datenqualität bzw. der mangelnden inhaltlichen Interpretation der Daten durch die PROVISO MitarbeiterInnen. Hier eine Anhebung des Qualitäts- bzw. Serviceniveaus und damit eine effektivere Verwendung der PROVISO Produkte zu gewährleisten, sollte eine der prioritären Zielsetzungen von PROVISO für die verbleibende Projektlaufzeit sein, die auch von den PROVISO MitarbeiterInnen eine intensivere Auseinandersetzung mit den zur Verfügung gestellten Daten erfordern würde. Ein wichtiger Ansatzpunkt scheint in diesem Zusammenhang auch eine intensivere - gegebenenfalls auch institutionalisierte - bilaterale Zusammenarbeit zwischen den entsprechenden PROVISO MitarbeiterInnen und den jeweiligen Programmdelegierten, und damit eine stärkere Einbindung der Programmdelegierten in die Interpretation der Daten, zu sein.

\section{Öffentlichkeitsarbeit von PROVSIO}

Während das PROVISO Service ministeriumsintern mittlerweile für ein anerkanntes Qualitätssiegel steht ist die Sichtbarkeit von PROVISO in der breiteren Öffentlichkeit bzw. innerhalb der Forschungscommunity nicht sehr hoch. Vor allem begrenzte Kapazitäten seitens des PROVISO Service sowie die Notwendigkeit eines vertraulichen Umgangs mit den Daten sprechen dabei gegen eine zu starke „Bewerbung“ von PROVISO in der breiteren Fachöffentlichkeit. Dennoch scheint insbesondere im Bereich der externen Forschungsgruppen und Organisationen ein zusätzliches Interesse und auch Nutzerpotenzial des PROVISO Service zu bestehen (siehe auch oben). Insbesondere in Hinblick auf einzelnen Forschungsgruppen, wie beispielsweise Universitäten, größere Industriebetriebe oder auch außeruniversitäre Forschungseinrichtungen sollte dabei über Möglichkeiten einer kosteneffizienten zur Verfügung Stellung relevanter PROVISO Daten nachgedacht werden. Zum Teil hat ja eine diesbezügliche Kommunikation beispielsweise mit dem Universitätssektor bereits begonnen. Eine breitere, aber gezielte Ansprache weiterer potenzieller Kundengruppen von PROVISO würde nicht nur dazu beitragen, die Effektivität des PROVISO Service zu erhöhen, sondern PROVISO auch in der breiteren Fachöffentlichkeit zu einer stärkeren Sichtbarkeit und Anerkennung verhelfen.

\section{Organisatorische Einbettung von PROVISO}

Die organisatorische Einbettung von PROVISO in die Ministerialstruktur bringt einige Vorteile mit sich. Die Verfügbarkeit des Service "in-house“ ermöglicht vor allem den Programmdelegierten und der EU-Koordinationsabteilung einen raschen Zugriff auf die Informationen und dürfte auch die oben geforderte verstärkte interne Abstimmung bzw. Koordination zwischen den Delegierten und den PROVISO MitarbeiterInnen erleichtern. Insbesondere der geforderte vertrauliche Umgang mit den von der EU Kommission gelieferten Daten ist innerhalb des Ministeriums vergleichsweise unproblematisch zu handhaben. Längerfristig wäre jedoch zu überlegen, in wie weit das Potenzial einer Dienstleistungs- und Informationsstruktur wie PROVISO beispielsweise durch Ansiedlung in der Strategieabteilung der Forschungsförderungsgesellschaft (FFG) nicht besser ausgeschöpft werden könnte. Hier würde sich neben Synergien mit bestehenden bzw. im Aufbau begriffenen Datenbanken auch die Möglichkeit ergeben, PROVISO in einen breiteren Kontext mit anderen Forschungsförderungen (z.B. Basisprogramme, Thematische Programme etc.) zu setzten und damit die Basis für ein umfassendes österreichisches Technologie- Forschungsmonitoring zu schaffen, das neben den EU-Rahmenprogrammen auch andere nationale und internationale Förderprogramme berücksichtigt. 
ANHANG 


\section{Die Aufbereitung von Beteiligungsdaten im internationalen Kontext}

\section{Methodische Anmerkungen}

Im Rahmen der Evaluierung des österreichischen PROVISO Service war auch zu untersuchen, inwieweit in anderen europäischen Ländern ähnliche Dienstleistungsangebote existieren bzw. ob und in welcher Form die von der Europäischen Kommission zur Verfügung gestellten Daten zur Beteiligung von ForscherInnen und F\&E-Einrichtungen an den EU Rahmenprogrammen erfasst und aufbereitet werden. Hierzu wurden auf Basis der im Rahmen dieser Evaluierung durchgeführten Interviews Länder identifiziert, von welchen bekannt ist (bzw. von welchen angenommen wird), dass sie PROVISO ähnliche Dienstleistungen zur Verfügung stellen. Staaten, die die Daten in systematischer Art und Weise erfassen und damit der Implementierung eines Services wie PROVISO am nächsten kommen, sind:

- Finnland,

- die Niederlande,

- Irland,

- und Schweden.

Von diesen Ländern wurden kurze Fallstudien erstellt, die auf Telefoninterviews mit den jeweils für die nationalen Datenaufbereitungsdienste verantwortlichen Personen basieren. Als Gegenkontrolle wurde bezüglich der Handhabung der von der EU Kommission zur Verfügung gestellten Daten auch in Staaten nachgefragt, in denen kein derartiges Service angeboten wird. Einschränkend ist festzuhalten, dass die Analyse nur einen Teil der an den EU Rahmenprogrammen beteiligten Länder explizit abdeckt.

\section{Länderergebnisse}

Im Folgenden sollen nun die nationalen Systeme zur Aufbereitung von Beteiligungsdaten an den EU Rahmenprogrammen in Finnland, den Niederlanden, in Irland und in Schweden (sowie in verkürzter Form auch in Dänemark, England und Polen) beschrieben werden.

\section{Finnland}

Die Verwaltung und Aufbereitung der Daten zur finnischen Beteiligung an den EU Rahmenprogrammen liegt im Verantwortungsbereich von TEKES, einer zentralen finnischen Förderagentur, die auch als Anlaufstelle für Anfragen zu den Rahmenprogrammen fungiert und dem dortigen Wirtschaftsministerium unterstellt ist. Trotz der Zuordnung zum Wirtschaftsministerium agiert TEKES hinsichtlich der EU Rahmenprogramme weitgehend unabhängig, da das Ministerium hauptsächlich nur eine Aufsichtsfunktion gegenüber der Agentur innehat.

TEKES hat, ähnlich wie Österreich, für die Verwaltung der Beteiligungsdaten eine Accessdatenbank erstellt, die mittels eines einfachen Webinterface über Internet passwortgeschützt für einen eingeschränkten Personenkreis (Ministerien, NCPs, Programmdelegierte und ausgewählte MitarbeiterInnen von TEKES) zugänglich ist. Im Gegensatz zu Österreich gibt es bei TEKES keine zentrale Stabsstelle für die Erfassung und Wartung der Datenbestände, in der MitarbeiterInnen ausschließlich zu diesem Zweck angestellt sind. Die Erstellung von Auswertungen und die globale Datenpflege obliegen einer TEKES Mitarbeiterin und ihrer Assistentin, die beide diese Arbeit zusätzlich zu anderen, nicht damit in Verbindung stehenden Aktivitäten von TEKES ausführen.

Die Programmdelegierten und NCPs sind verpflichtet, Daten zu ihren Programmen über das Webinterface in das System einzugeben, sobald entsprechende neue Informationen auf CIRCA veröffentlicht werden. Erfasst werden somit alle Programme, unabhängig vom Aufwand für die Datenaufbereitung. Den Delegierten und NCPs ist es freigestellt, für die Erfassung zusätzliches Personal anzuwerben. In Ausnahmefällen kann auch TEKES unterstützend eingreifen und, aufwandabhängig, Werkvertragsstudenten für die Datenerfassung hinzuziehen. In die Daten- 
bank werden auch, soweit verfügbar, Detaildaten zu den Antragstellerlnnen eingespielt. Eine Datenabfrage ist u.a. an Hand folgender Kriterien möglich: Titel des Antrags, Thema, Instrument, beantragte und bewilligte Mittel, Akronym, Land des Koordinators, Beteiligung anderer Länder (d.h. die entsprechenden Ländernamen), Namen der beteiligten Institutionen, Namen der ProjektleiterInnen und beteiligten ForscherInnen sowie deren Kontaktdaten. Erfasst werden auch nicht erfolgreiche Anträge.

Standardmäßig werden nur zwei Produkte erstellt:

- Ein habjährig erscheinender Gesamtbericht über die finnische Beteiligung: Dieser besteht aus einer Kurzzusammenfassung (1-seitig), einer Exceldatei mit den relevanten Daten und vier Folien mit Übersichtsgrafiken.

- Ein jährlich erscheinender Foliensatz zu finnischen Kooperationsaktivitäten im 6. EU Rahmenprogramm: Hierbei handelt es sich um ein neues Produkt, das erstmals 2004 publiziert wurde.

Werden Spezialauswertungen gewünscht, wenden sich die Ministerien, NCPs und Delegierten direkt an TEKES, welches die Anfragen bearbeitet. Die Ergebnisse werden dann formlos per EMail verschickt. Eine direkte Durchführung der Anfrage über das Extranet ist indes nicht möglich, da die entsprechende Suchfunktion keine komplexen Operationen zulässt. Ein wesentlicher Unterschied zu Österreich ist, dass externe Einrichtungen und Interessensgruppen keine Anfragen stellen dürfen bzw. können. Pro Jahr werden etwa 100 Anfragen bearbeitet.

\section{Die Niederlande}

In den Niederlanden besteht mit dem EG-LIAISON Office eine zentrale Anlaufstelle für alle Angelegenheiten, die mit dem 6 . EU Rahmenprogramm in Verbindung stehen. Infolgedessen ist EG-LIAISON auch mit der Aufbereitung der Daten der EU Kommission zur niederländischen Beteiligung an den EU Rahmenprogrammen betraut, bietet darüber hinaus aber auch Beratungsdienstleistungen an.

EDV-mäßig wird in den Niederlanden auf einen Mix verschiedener Tools zurückgegriffen. U.a. wird, in Lizenz, auch das INNOMAN System der Abteilung EIP in der FFG verwendet. Die Aufbereitung der Kommissionsdaten erfolgt im Rahmen der anderen Tätigkeiten von EGLIAISON, d.h. es gibt keine Stabsstelle, die ausschließlich ein PROVISO ähnliches Service anbietet. Eingespielt wird nur ein Teil der von der EU Kommission bereitgestellten Daten, nämlich ausschließlich jene Programminformationen, die maschinell leicht verarbeitbar sind. Die schlechte Datenqualität ist auch in den Niederlanden ein Thema und führt derzeit zu breiten Diskussionen darüber, inwieweit ein derartiges Service für die Zukunft überhaupt aufrechterhalten werden kann. Eine entsprechende Entscheidung ist noch nicht gefallen.

Standardmäßig werden folgende Produkte erstellt:

- Ein Jahresgesamtbericht,

- Programmberichte für jedes Programm,

- Callberichte für jeden Call.

Für jene Programme, bei denen Detaildaten zu den Einreicher/innen in vernünftiger Form erfasst werden können, werden auch Spezialanfragen, z.B. für das Wirtschaftsministerium, bearbeitet. Diese sind vertraulich, während die Standardprodukte öffentlich zugänglich sind. 


\section{Irland}

Irland versucht, einen Großteil der in den CIRCA Dateien dokumentierten irischen Beteiligungsdaten zu erfassen. Die entsprechenden Tätigkeiten und Kompetenzen sind zentral bei FORFAS (einer Einrichtung, die für diverse Entscheidungsgremien und Räte Statistik- und Recherchedienstleistungen zur Verfügung stellt) gebündelt. Innerhalb von FORFAS ist eine Person für die Aufbereitung der Daten zuständig, wie in den anderen Ländern aber nicht ausschließlich hierfür abgestellt.

Anders als in Finnland und ähnlich wie in Österreich leiten die NCPs/Delegierten ihre CIRCA Daten an FORFAS weiter, die wiederum (unter Zuhilfenahme von zusätzlichen Arbeitskräften) die Daten in eine realtimefähige ORACLE Datenbank eingeben. Bei Programmen, bei denen die Datenqualität schlecht ist, dauert dies entsprechend länger. Die ORACLE Datenbank bildet die Basis für folgende Standardprodukte:

- $\quad$ Ein Jahresbericht zur Performance Irlands im 6. EU-Rahmenprogramm: Dieser ist sehr detailliert und bietet einen Vergleich zwischen einzelnen Universitäten, Technologiehochschulen und Unternehmen. Im Bericht wird u.a. nach Instrumenten und nach den Besitzverhältnissen der beteiligten Unternehmen (national - international) differenziert. Außerdem gibt es einen Abschnitt zu Kooperationsaktivitäten zwischen Wirtschaft und Wissenschaft.

- Monatsberichte zur Beteiligung Irlands am 6. EU Rahmenprogramm: Diese werden für die Regierung und das Wirtschaftsministerium erstellt, aber auch an die NCPs verschickt. Sie sind weniger umfangreich als der Jahresbericht.

Daneben können auch Spezialanfragen informell bearbeitet werden. Die Berichte, die per se nicht der breiten Öffentlichkeit zugänglich gemacht werden, verstehen sich als Motivations- und Diskussionsgrundlage für alle sich am 6. EU Rahmenprogramm beteiligenden akademischen Institutionen und werden auch sämtlichen Universitäten, Hochschulen und Beratungseinrichtungen zur Kenntnis- und/oder Stellungnahme (als PDF Files) zugesandt. In speziellen „Coordination Group Meetings“ werden die Ergebnisse von den Akteuren erörtert. Externe Anfragen werden von FORFAS nicht beantwortet, allerdings soll es bislang auch noch keine externen Anfragen gegeben haben.

\section{Schweden}

Das 1992 gegründete EU/FoU-rådet (engl: „Swedish EU R\&D Council“) fungiert als One-Stop Shop für die EU Rahmenprogramme in Schweden. Neben Beratungsdienstleitungen ist EU/FoU auch für die Aufbereitung der Informationen zur Beteiligung schwedischer Forscher/innen am 6. EU Rahmenprogramm zuständig, wofür ein einzelner Mitarbeiter abgestellt wurde.

EU/FoU erhält sämtliche Daten der EU Kommission, die Schweden zur Verfügung gestellt werden, nutzt aber, auf Grund des Aufwandes, lediglich die publizierten CD ROMs der EU Kommission für Auswertungen. CIRCA Daten werden nur verwendet, um sich einen qualitativen Überblick zu verschaffen. Damit kann Schweden auch als „Übergangsland“ zwischen jenen Ländern, die einen mehr oder weniger aktiven PROVISO ähnlichen Service auf CIRCA Datenbasis anbieten und jenen Staaten, die keine oder nur ad hoc Auswertungen von Kommissionsdaten vornehmen, angesehen werden. Nachteil der CD-ROM Lösung ist auf jeden Fall die geringere Aktualität der dort gespeicherten Informationen.

Berichte zur Beteiligung Schwedens am 6. EU Rahmenprogramm werden erstellt, sobald eine neue CD ROM verfügbar ist, d.h. im Normalfall mehrmals pro Jahr. Nachfrage nach diesen Berichten besteht vor allem auf Seiten der Regierung und des Ministeriums sowie des schwedischen statistischen Zentralamtes. Letzteres verwendet die Auswertungen für die jährlichen statistischen Jahrbücher. Anfragen von NCPs und Programmdelegierten sind selten, da diese ihre Auswertungen (auf CIRCA Basis) selbst vornehmen. 


\section{Andere Staaten}

In anderen Staaten gibt es nur sehr wenige koordinierte Tätigkeiten hinsichtlich einer Aufbereitung der nationalen Beteiligungsdaten am 6. EU Rahmenprogramm. In England liegt die Verantwortung beim NCP Koordinator, der aber - wie in Schweden - auf Grund des hohen Aufwandes nur mit den CD ROM Daten arbeitet. Bei größeren Ländern muss allerdings auch festgehalten werden, dass auf Grund der höheren Anzahl an Anträgen auch der Aufwand proportional steigt. In Dänemark wird seit letztem Jahr aus CIRCA und CD ROM Daten vom Wirtschaftsministerium ein Jahresbericht erstellt, der nur Text und überblicksmäßige Eckdaten enthält. In Polen wird der Aufbau eines zentralen PROVISO-ähnlichen Systems ins Auge gefasst, wobei man aber über improvisierte Lösungen (zum Teil mit Datenbanken, zum Teil mit Excelfiles) nicht hinauskommt, aber zumindest in der Lage war, einen Jahresbericht zu produzieren. Kritisiert wird auch hier die schlechte Datenqualität der Kommissionsfiles.

Fazit

Innerhalb der analysierten Länder dürfte Österreich über das mit Abstand umfangreichste System zur Aufbereitung und Auswertung von Daten zur Beteiligung am 6. EU Rahmenprogramm verfügen. Jene wenigen Länder, die ähnliche Services implementiert haben, stellen hierfür deutlich geringere Ressourcen zur Verfügung und nehmen in der Folge entsprechende Einschränkungen im Leistungsangebot in Kauf. Die meisten Länder führen nur ad hoc Auswertungen auf Basis von älteren Überblicksdaten durch. Inwieweit Österreich vom höheren Aufwand profitiert, ist objektiv schwer abzuschätzen. Den Angaben der Delegierten zufolge hat man gegenüber Kolleg/innen aus anderen Ländern einen Informationsvorsprung, kann schneller strategische Entscheidungen treffen und gegenüber der Kommission in Teilbereichen mehr Druck ausüben (z. B. kann auf Ungleichbehandlungen in verschiedenen Programmen hingewiesen und fragwürdige Begründungen der Kommission hierfür erörtert werden). In wie weit sich dies auf die Erfolgsquote österreichischer Beteiligungen an den EU-Rahmenprogrammen auswirkt ist hingegen schwer zu eruieren, der primäre Nutzen des PROVISO Service dürfte allerdings eher in der Verfügbarkeit eines umfassenden Technologie- und Forschungsmonitoring als Grundlage für die nationale und internationale Strategieentwicklung und Politikberatung sowie eine entsprechende Öffentlichkeitsarbeit liegen, als in einer Verbesserung der Position der nationalen Programmdelegierten in den entsprechenden Programmausschusssitzungen in Brüssel. 


\section{Liste der Interviewpartner/innen}

\begin{tabular}{|c|c|c|c|}
\hline & Name & Institution & Funktion \\
\hline \multicolumn{4}{|c|}{ Auftraggeber } \\
\hline 1 & Dr. Christian Seiser & BMBWK & Leiter der Abteilung für EU-Forschungskoordination \\
\hline 2 & Dr. Rupert Pichler & BMVIT & $\begin{array}{l}\text { Leiter der Abteilung Forschungs- und Technologie- } \\
\text { förderung }\end{array}$ \\
\hline \multicolumn{4}{|c|}{ Programmdelegierte } \\
\hline 3 & Mag. Markus Pasterk & BMBWK & $\begin{array}{l}\text { Genomik und Biotechnologie im Dienste der } \\
\text { Gesundheit }\end{array}$ \\
\hline 4 & Dr. MBA Irene Gabriel & BMBWK & $\begin{array}{l}\text { Nachhaltige Entwicklung, globale Veränderungen } \\
\text { und Ökosysteme }\end{array}$ \\
\hline 5 & Mag. Martina Hartl & BMBWK & $\begin{array}{l}\text { Bürger und Staat in der Wissensgesellschaft / } \\
\text { Wissenschaft und Gesellschaft }\end{array}$ \\
\hline 6 & Dr. Daniel Weselka & BMBWK & $\begin{array}{l}\text { Nanontechnologien, intelligente Materialien und } \\
\text { neue Produkte, EURATOM, JRC Aktivitäten }\end{array}$ \\
\hline 8 & $\begin{array}{l}\text { Mag. Christian Drakulic (iV. } \\
\text { Dr. Andreas DORDA) }\end{array}$ & BMVIT & Transport \\
\hline 9 & Mag. Eva Gottmann & BMBWK & Ausbildung und Mobilität von Forscher/innen \\
\hline 10 & Mag. Michael Wiesmüller & BMVIT & Technologien für die Informationsgesellschaft \\
\hline 11 & Dipl. Ing. Dr. Gerald Vones & BMWA & $\begin{array}{l}\text { Nachhaltige Entwicklung, globale Veränderungen } \\
\text { und Ökosysteme für energiebezogene Aspekte }\end{array}$ \\
\hline 12 & Dr. Anita Silmbrod & BMLFUW & Lebensmittelqualität und -sicherheit \\
\hline \multicolumn{4}{|c|}{ PROVISO MitarbeiterInnen } \\
\hline 13 & Mag. Michaela Topolnik, MA & $\begin{array}{l}\text { RFT (ehemals } \\
\text { PROVISO) }\end{array}$ & $\begin{array}{l}\text { Geschäftsstelle (ehemalige Projektleiterin von } \\
\text { PROVISO) }\end{array}$ \\
\hline 14 & Mag. Günter Dinhobl & PROVISO & Projektleiter \\
\hline 15 & DI Margit Ehardt-Schmiederer & PROVISO & Stv. Projektleiterin \\
\hline 16 & Mag. Tamara Coja & PROVISO & PROVISO Mitarbeiterin \\
\hline \multicolumn{4}{|c|}{ Beratung- und Betreuungseinrichtungen } \\
\hline 17 & Mag. Andrea Fenz & APS & Geschäftsführung \\
\hline 18 & Mag. Birgit Steininger & FFG-EIP & $\begin{array}{l}\text { Leiterin der Abteilung Biowissenschaften und - } \\
\text { technologien }\end{array}$ \\
\hline 19 & Dr. Wolfram Rieneck & BEP & Projektmanager EU Forschungsprojekte \\
\hline 20 & DI Manfred Ruhmer & CATT Linz & $\begin{array}{l}\text { zuständig für EU-Forschungsrahmenprogramme, } \\
\text { Nationale/Regionale F\&E-Förderung }\end{array}$ \\
\hline 21 & Mag. Ferdinand Steger & ITG Salzburg & $\begin{array}{l}\text { Abteilung Forschung und Entwicklung } \\
\text { 6. EU-Rahmenprogramm }\end{array}$ \\
\hline \multicolumn{4}{|c|}{ Stakeholder } \\
\hline 22 & Ursula Suda & BMBWK & $\begin{array}{l}\text { Evaluierung von Forschungstätigkeiten, Controlling } \\
\text { und Dokumentation }\end{array}$ \\
\hline 23 & Mag. Harald Hochreiter & $\begin{array}{l}\text { Max F. Perutz } \\
\text { Laboratories }\end{array}$ & Geschäftsführer \\
\hline 24 & Mag. Klaus Zinöcker & WWTF & Mitarbeiter \\
\hline
\end{tabular}




\begin{tabular}{|c|c|c|c|}
\hline \multicolumn{4}{|c|}{ Für den internationalen Vergleich } \\
\hline 25 & Maija Hakkarinen & TEKES (FIN) & EU R\&D Adviser \\
\hline 26 & Imelda Lambkin & FORFAS (IR) & Researcher \\
\hline 27 & Eric van Pelt & EG-Liaison (NL) & Mitarbeiter \\
\hline 28 & Anna Pytko & IFTR-PAS(PL) & $\begin{array}{l}\text { Member of Programme Committee for FP5 and } \\
\text { FP6 }\end{array}$ \\
\hline 29 & Robin Hurst & $\begin{array}{l}\text { Department of } \\
\text { Trade and } \\
\text { Industry (UK) }\end{array}$ & FP6 National Coordinator \\
\hline 30 & Hans Bergmann & EU/FoU (SE) & Mitarbeiter, zuständig für EU Beteiligungsdaten \\
\hline 31 & Jan Corner-Walken & Eurocenter (DK) & Innovation NCP \\
\hline
\end{tabular}

